\title{
Applicability of the convergence-confinement method to full-face excavation of circular tunnels with stiff support system
}

\author{
Manuel DE LA FUENTE ${ }^{1,2}$, \\ Reza TAHERZADEH ${ }^{1}$, \\ Jean SULEM ${ }^{2}$, \\ Xuan-Son NGUYEN ${ }^{1}$, \\ Didier SUBRIN ${ }^{3}$ \\ ${ }^{1}$ Tractebel Engie, 5 Rue du 19 Mars 1962, 92622 Gennevilliers, France \\ ${ }^{2}$ Laboratoire Navier/CERMES, Ecole des Ponts ParisTech, IFSTTAR, CNRS, Université \\ Paris-Est, 6, 8 Avenue Blaise Pascal, 77455 Marne la Vallée, France, Email: \\ jean.sulem@enpc.fr, Phone : +33164153545 \\ ${ }^{3}$ Centre d'Etudes des Tunnels (CETU), 25 Avenue François Mitterrand, 69674 Bron Cedex 1, \\ France
}

\begin{abstract}
:
The ConVergence-ConFinement (CV-CF) method is widely used in conventional tunneling at a preliminary stage of the design. In this method, the rock-support interaction is simplified by means of a two-dimensional plane-strain assumption. However, when the ground exhibits large deformation and/or when the support is very stiff and installed close to the tunnel face, the results obtained with the CV-CF method may significantly differ from those obtained using 3D numerical computations. The strong interaction taking place between the rigid lining and the rock mass is not considered in the most common use of the CV-CF method. Some improvements of the CV-CF method as the so-called implicit methods have been developed in order to better account for this interaction.

In this paper, the applicability of the CV-CF methods is discussed for full face excavation tunneling with a stiff support system. An in-depth comparison between plane-strain closed form solutions and numerical results which properly accounts for the 3D effects at the vicinity of the tunnel face is carried out. The range of application of the different approaches of the CV-CF method is discussed. Finally, some simple empirical formula which can be used in preliminary design for a large range of ground conditions are proposed.
\end{abstract}

Keywords: Convergence-confinement method; Tunneling; Single shield TBM; Groundlining interaction. 


\section{List of symbols}

$x \quad$ Distance to the tunnel face

$R \quad$ Tunnel radius

D Tunnel diameter

$d \quad$ Distance of support/lining installation from the tunnel face

$x_{f} \quad$ Distance between the edge of the lining and the tunnel face (unsupported length)

$s \quad$ Step round length in the numerical simulations

$e \quad$ Thickness of the support or the lining

$R_{o} \quad$ Outer radius of a lining

$R_{i} \quad$ Inner radius of a lining

$\lambda(x)$ Deconfining rate which depends on the distance to the advancing face $x$

$c \quad$ Cohesion of the ground

$\phi \quad$ Friction angle of the ground

$\psi \quad$ Dilatancy angle of the ground

$K_{p} \quad$ Friction parameter of the ground

$\beta \quad$ Dilatancy parameter of the ground

$v \quad$ Poisson's ratio of the ground

E Young's modulus of the ground

$G \quad$ Shear modulus of the ground

$\mathrm{v}_{l} \quad$ Poisson's ratio of the support or the lining

$E_{l} \quad$ Young's modulus of the support or the lining

$G_{l} \quad$ Shear modulus of the support or the lining

$N \quad$ Stability number

$R_{p l} \quad$ Plastic radius (unsupported opening)

$k_{s n} \quad$ Normal stiffness of the support

$K_{s n} \quad$ Normal stiffness of a lining

$u(x) \quad$ Radial displacement at the tunnel wall which depends on the distance to the advancing face $x$ (unsupported opening)

$\bar{u}(x) \quad$ Radial displacement at the tunnel wall which depends on the distance to the advancing face $x$ (supported opening)

$p_{f} \quad$ Fictitious pressure applied to the tunnel boundary (in order to account for the influence of the tunnel face)

$\sigma_{0} \quad$ Initial isotropic stress state in the ground

$\sigma_{c} \quad$ Uniaxial compression strength

$p_{s} \quad$ Radial pressure acting upon the outer boundary of the lining

$\sigma_{\max } \quad$ Maximal hoop stress developed in the support or the lining at the state of equilibrium

$\chi \quad$ Homothetic ratio in the Self Similarity Principle

..., ${ }^{*} \quad$ Normalized parameter/variable

$\ldots$, el Elastic parameter/variable

$\cdots, p l \quad$ Elastoplastic parameter/variable 


\section{Acknowledgements}

This work is part of the PhD thesis of the first author, carried out at Ecole des Ponts ParisTech in partnership with Tractebel ENGIE and CETU (Centre d'Études des Tunnels). The authors wish to thank ITASCA for supporting the first author through the Itasca Education Partnership Program (IEPP). 


\section{Introduction}

The ConVergence-ConFinement ( $\mathrm{CV}-\mathrm{CF})$ method is a basic and largely used tool for the preliminary design of underground support structures. Based on the analysis of stresses and strains around a circular tunnel, it provides an insight into the interaction between the support and the ground by means of a plane-strain model of the tunnel excavation. This technique is based on three different curves which are combined in order to calculate the equilibrium state between the support and the ground. These curves are the Longitudinal Displacement Profile (LDP), the Ground Reaction Curve (GRC) and the Support Confining Curve (SCC). Limitations of the CV-CF method have been discussed (e.g. Eisenstein and Branco, 1991) and extension have been proposed for shallow tunnels, non-circular cross sections, reinforced rocks (e.g. GonzalezNicieza et al., 2008; Oreste, 2009; Wong et al., 2006; Tran et al., 2015) and to account for timedependent effects (Sulem et al., 1987) or seepage forces (Lee et al., 2007).

The development of CV-CF method is based on the assumption that an intrinsic GRC effectively exists. However, when a stiff lining is placed immediately near the advancing face, the GRC is affected by the presence of the lining (Cantieni and Anagnostou, 2009). Tunneling is indeed an inherently three-dimensional (3D) mechanical problem and as a consequence, the spatial effects that take place in the vicinity of the tunnel face are not properly simulated with a two-dimensional (2D) plane-strain model. Therefore, the calculated state of equilibrium differs between 2D and 3D analyses. This discrepancy is even stronger when large deformations take place (Cantieni and Anagnostou, 2009). Many other practical limitations of the 2D analysis have been highlighted. Schürch and Anagnostou (2012) have discussed the applicability limits of the closed-form GRC solution for a circular tunnel excavated in an isotropic ground when the rotational symmetry of the problem in terms of stress state and section shape is violated. Vlachopoulous and Diederichs (2014) have shown that for tunnels with sequenced support installation steps or non-isotropic stresses, 3D analyses are necessary.

The present paper deals with the design of circular tunnels with stiff support system focusing on the typical example of tunnels excavated with a single shield TBM in rock masses. This topic was already addressed by Ramoni et al. (2011) for tunnels excavated in squeezing conditions with a single shield TBM. These authors have provided a series of design charts for the estimation of the maximal load exerted on the segmental linings considering the effect of the TBM characteristics (stiffness, conicity, backfilling). In the present study, the applicability of the different approaches of the CV-CF method is discussed considering a large range of ground properties and various excavation methods. Finally, some empirical relationships are proposed for use within preliminary design of tunnels excavated with single shield TBM.

\section{The CV-CF method}

\subsection{Principle of the method}

The CV-CF method has been originally developed for full face circular tunnels excavated in a homogeneous ground in isotropic stress conditions where the gravity effects can be disregarded (e.g. AFTES, 2002). The combination of GRC, LDP and SCC allows to obtain the equilibrium state as will be explained in the subsequent sections. 
The 3D problem is simplified by means of a 2D plane-strain assumption where the tunnel excavation is simulated by a progressive reduction of a 'fictitious' internal support pressure $p_{f}$ applied at the tunnel wall

$$
p_{f}=(1-\lambda) \sigma_{0}
$$

where $\sigma_{0}$ is the initial stress state which is here assumed isotropic and $\lambda$ is the deconfining rate.

The idea of employing a deconfining rate $\lambda$ in order to simulate the support effect of the face advance was introduced by Panet and Guellec (1974). It takes the value of 0 at the initial state and grows until reaching the value of 1 when the tunnel is completely excavated.

\subsection{Ground reaction curve (GRC)}

The GRC is the relationship between the progressive reduction of the fictitious pressure and the radial displacement of the tunnel boundary $u(x)$. Equation 2 gives the expression of the GRC for a linear elastic ground.

$$
u(x)=\lambda(x) u(\infty)_{e l}
$$

where $u(\infty)_{e l}$ is the radial displacement at the tunnel wall for an elastic tunnel far away from the tunnel face and is expressed as

$$
u(\infty)_{e l}=\frac{\sigma_{0} R}{2 G}
$$

where $R$ is the radius of the tunnel and $G$ is the elastic shear modulus.

The expression of the GRC for an elastoplastic ground when a Mohr-Coulomb yield criterium is adopted can also be expressed in terms of $\lambda$ (Panet, 1995)

$$
\begin{gathered}
u(x)=R \frac{(1+v)}{E}\left(C_{1}+C_{2}\left(\frac{R}{R_{p l}}\right)^{K_{p}-1}+C_{3}\left(\frac{R_{p l}}{R}\right)^{\beta+1}\right) \\
C_{1}=-(1-2 v)\left(\sigma_{0}+H\right) \\
C_{2}=\left(\frac{(1-v)\left(1+\beta K_{p}\right)}{K_{p}+\beta}-v\right) \frac{2\left(\sigma_{0}+H\right)}{K_{p}+1} \\
C_{3}=2(1-v) \frac{\left(K_{p}-1\right)\left(\sigma_{0}+H\right)}{K_{p}+\beta}
\end{gathered}
$$




$$
\begin{gathered}
R_{p l}=\left[\frac{2\left(\sigma_{0}+H\right)}{K_{p}+1} \frac{R^{K_{p}-1}}{(1-\lambda) \sigma_{0}+H}\right]^{\frac{1}{K_{p}-1}} \\
H=\frac{c}{\tan \phi} \\
\beta=\frac{1+\sin \psi}{1-\sin \psi} \\
K_{p}=\frac{1+\sin \phi}{1-\sin \phi}
\end{gathered}
$$

where $R_{p l}$ is the ultimate plastic radius, $\phi$ is the friction angle, $\psi$ is the dilatancy angle, $c$ is the cohesion, $E$ is the Young's modulus, $v$ is the Poisson's ratio, $\beta$ is the dilatancy parameter and $K_{p}$ is the friction parameter.

\subsection{Support Confining Curve (SCC)}

The SCC describes the mechanical response of the support/lining. For an elastic support, assuming axial symmetry of the applied loads and of the support geometry, the relationship between the radial displacement of the wall at the outer face of the support $(\bar{u}(x)-\bar{u}(d))$ and the radial inward pressure exerted by the ground $p_{s}$ acting upon the support is given by

$$
\frac{\bar{u}(x)-\bar{u}(d)}{R}=\frac{p_{s}}{k_{s n}}
$$

where $\bar{u}(x)$ is the radial displacement at the tunnel wall which depends on the distance to the advancing face $x$ for a supported opening, $d$ is the distance of support/lining installation and $k_{s n}$ is the elastic normal stiffness of the support. For a sprayed concrete support/lining of thickness $e$, the stiffness is obtained from the thin shell theory, (equation 13).

$$
k_{s n}=\frac{E_{l}}{1-v_{l}^{2}} \frac{e}{R}
$$

where $E_{l}$ and $v_{l}$ are the Young modulus and the Poisson ratio of the sprayed concrete respectively.

However, thin shells theory can only be applied when $e<R / 20$ (Flügge 1960). De Labriolle (2017) has shown that adopting a thin shell approach for a thick support/lining, induces important errors. For TBM tunneling, the segmental lining thickness is generally in the order of $R / 10$. Therefore, resorting to the thick shell theory the normal stiffness of a thick lining $K_{s n}$ is given by:

$$
K_{s n}=\frac{2 G_{l}\left(R_{o}^{2}-R_{i}^{2}\right)}{\left(1-2 \mathrm{v}_{l}\right) R_{o}^{2}+R_{i}^{2}}
$$


where $G_{l}$ is the shear modulus of the concrete lining, $R_{o}$ and $R_{i}$ are the outer and the inner radius of the lining respectively.

In the lining, the maximal hoop stress $\sigma_{\max }$ takes place at the inner face.

$$
\sigma_{\max }=\frac{2 p_{s} R_{o}^{2}}{\left(R_{o}^{2}-R_{i}^{2}\right)}
$$

The equilibrium is obtained at the intersection of the GRC and the SCC as shown in Fig1. However, this result depends on the evaluation of $\bar{u}(d)$ (obtained from the LDP).

\subsection{Longitudinal Displacement Profile (LDP)}

The closed-form expression of the GRC for an unsupported tunnel can be established for various constitutive models. The SCC can be calculated for most of the existing supports. However, the LDP, which relates the displacement around the tunnel and the distance to the advancing face, is commonly expressed by using empirical formulas derived from the results of axisymmetric numerical simulations. Depending on the underlying assumptions for the LDP (which determines the value of $\bar{u}(d)$ ), we can distinguish between various CV-CF approaches.

\subsubsection{The Classical CV-CF approach}

Within the classical approach, the GRC and the LDP are considered to be intrinsic curves of the ground and they are assumed to be independent from the support behavior. As a consequence, $\bar{u}(d)$ is equal to the radial displacement of the unsupported tunnel wall at the instant of the support installation $u(d)$ (Fig1).

Fig1. Schematic representation of the curves employed in the CV-CF method

The LDP of an unsupported tunnel excavated in an elastic ground can be written as follows:

$$
u(x)=a(x) u(\infty)_{e l}
$$

where $x$ is the distance to the tunnel face, $a(x)$ is a dimensionless shape function

$$
a(x)=\frac{u(x)}{u(\infty)_{e l}}
$$

A commonly used expression which describes the shape function is given by Panet (1995) as obtained by fitting the results obtained from axisymmetric numerical computations

$$
a_{1}(x)=\alpha_{0}+\left(1-\alpha_{0}\right)\left(1-\left[\frac{m R}{m R+x}\right]^{2}\right)
$$

Typical values of the parameters are $\alpha_{0}=0.25$ and $m=0.75$ or $\alpha_{0}=0.27$ and $m=0.84$.

Corbetta et al. (1991) have proposed a different expression for the shape function: 


$$
a_{2}(x)=0.29+0.71\left(1-e^{\left(-1.5\left(\frac{x}{R}\right)^{0.7}\right)}\right)
$$

Both expressions $a_{1}(x)$ and $a_{2}(x)$ lead to very similar LDP for an elastic ground.

The first empirical expression for the shape function for an elastoplastic ground was proposed by Panet and Guénot (1983)

$$
b(x)=1-\left[\frac{0,84 R_{p l}}{0,84 R_{p l}+x}\right]^{2}
$$

which leads to the following expression for the LDP:

$$
u(x)=u(0)+\left(1-\left[\frac{0,84 R_{p l}}{0,84 R_{p l}+x}\right]^{2}\right)(u(\infty)-u(0))
$$

where $u(0)$ is the radial displacement at the tunnel wall at the face.

These authors also proposed a graphical approach which enables to obtain the deconfining parameter $\lambda$ depending on the so-called stability number $N$ (equation 22) and on the distance to the tunnel face $x$.

$$
N=\frac{2 \sigma_{0}}{\sigma_{c}}
$$

where $\sigma_{c}$ is the uniaxial compression strength.

The formulation for the elastic ground behavior can be extended to an elastoplastic ground by using the Self Similarity Principle (SSP) as introduced by Corbetta et al. (1991). The principle of the method is to apply a homothetic transformation to the LDP of a tunnel excavated in an elastic ground in order to get the one for an elastoplastic (.)pl ground. The homothetic ratio $\chi$ is given by:

$$
\chi=\frac{u(\infty)_{p l}}{u(\infty)_{e l}}
$$

From equations 18 (or 19) the LDP based on the SSP takes the following form:

$$
u(x)=\chi a\left(\frac{x}{\chi}\right) \frac{\sigma_{0} R}{2 G}
$$

For commonly encountered ground conditions $(N \leq 5)$, the obtained LDP gives acceptable results.

A more robust formulation for the LDP was proposed by Vlachopoulous and Diederichs 
(2009). They consider that the LDP is a function of tunnel radius and the extent on the ultimate plastic radius. According to these authors, the proposed expression is appropriate for modelling large convergences when $N>5$.

$$
\left\{\begin{array}{cr}
u(0)^{*}=\frac{u(0)}{u(\infty)}=\frac{1}{3} e^{-0.15 R p l^{*}} & \text { for } x^{*}=\frac{x}{R}=0 \text { (at the face) } \\
u(x)=u(\infty) u_{0}^{*} e^{x^{*}} & \text { for } x^{*}=\frac{x}{R} \leq 0 \text { (ahead of the face) } \\
u(x)=u(\infty)\left(1-\left(1-u_{0}^{*}\right) e^{\left.\frac{-3 x^{*}}{2 R_{p l}{ }^{*}}\right)}\right. & \text { for } x^{*}=\frac{x}{R}>0 \text { (in the tunnel) }
\end{array}\right.
$$

where $R_{p l}{ }^{*}$ is the normalized plastic radius $\left(R_{p l}{ }^{*}=\frac{R_{p l}}{R}\right), x^{*}$ is the normalized distance to the face and $u(0)^{*}$ is the normalized radial displacement around the tunnel boundary at the tunnel face for an unsupported opening.

Assuming that $\bar{u}(d)$ is equal to $u(d)$ can induce significant errors when a stiff lining is installed close to the face. In order to account for the ground-lining interaction, some authors have proposed to enhance the classical CV-CF method by resorting to the so-called implicit methods. In these methods, the effect of the support stiffness is taken into account in the evaluation of $\bar{u}(d)$.

\subsubsection{The "new implicit CV-CF approach" of Bernaud \& Rousset}

Bernaud and Rousset $(1992,1994)$ have proposed the "new implicit CV-CF approach". This method modifies the shape of the LDP of a supported tunnel by applying a transformation to the $x$-axis. This transformation is a mathematical affinity which consists in squeezing the axis with a ratio which depends on the support stiffness. The new shape function $b^{s}(x)$ for the LDP can be obtained from the shape function of the unsupported LDP $b(x)$ (equation 21) as:

$$
b^{s}(x)=b(\alpha x) \text { with } \alpha=\alpha\left(k_{s n}{ }^{*}\right)
$$

Assuming an elastic behavior of the ground, Bernaud and Rousset (1992) have proposed an empirical expression for $\alpha\left(k_{s n}{ }^{*}\right)$ by fitting axisymmetric finite element computations.

$$
\begin{gathered}
\alpha\left(k_{s n}{ }^{*}\right)=1+ \\
-0.635 k_{s n}{ }^{*}-0.0293 k_{s n}{ }^{* 2}+0.781 .10^{-3} k_{s n}{ }^{* 3} \\
-0.64 .10^{-5} k_{s n}{ }^{* 4}
\end{gathered}
$$

In the case of a Mohr-Coulomb elasto-plastic behavior, the expression for $\alpha\left(k_{s n}{ }^{*}\right)$ is

These expressions are valid for $k_{s n}{ }^{*} \leq 7.2$ where $k_{s n}{ }^{*}$ is the normalized stiffness of the support: 


$$
k_{s n}^{*}=\frac{k_{s n}}{E}
$$

The implicit relationship between $\bar{u}(d)$ and the ground displacement around a supported opening at the equilibrium state $\bar{u}(\infty)$ is given by:

$$
\bar{u}(d)=u(0)+b^{s}(d)(\bar{u}(\infty)-u(0))
$$

Bernaud and Rousset (1992) proposed the following empirical expression for the calculation of the convergence at the tunnel face $u(0)$ if the behavior of the ground is elastic:

$$
u(0)=0.27 \frac{\sigma_{0} R}{2 G}
$$

whereas if the ground is elastoplastic, $u(0)$ will depend on the stability number $N$ :

$$
u(0)=R\left(0.17153+0.12747 N-0.027275 N^{2}\right) u(\infty)
$$

According to the authors, the "new implicit method" can be applied if $N \leq 5$.

\subsubsection{The implicit CV-CF approach of Nguyen-Minh \& Guo}

The implicit method of Nguyen-Minh and Guo (1996) is more commonly used. These authors have proposed a general relationship between $\bar{u}(d)$ and $\bar{u}(\infty)$. In this method, a reduction factor which implicitly depends upon $\bar{u}(\infty)$ is applied to the radial displacement of the tunnel wall $u(d)$ at the instant of installation of the lining in order to obtain $\bar{u}(d)$

$$
\bar{u}(d)=\Phi\left(\frac{\bar{u}(\infty)}{u(\infty)}\right) u(d)
$$

and $\Phi$ is an empirical function of polynomial form

$$
\Phi(x)=0,55+0,45 x-0,42(1-x)^{3}
$$

According to the authors, this method can be applied if $N \leq 5$ for any value of the support stiffness.

The application of the different methods is summarized in Tab1.

\section{Tab1. Summary for the application of the CV-CF methods}

\section{Applicability of the CV-CF methods}

Conventional tunnel excavation may be undertaken using different support methods: the socalled 'heavy method' makes use of a stiff primary lining whereas the 'light method' makes 
use of a deformable support system, which may even be allowed to yield so as to tackle difficult ground conditions such as within high overburden and squeezing rock behavior. In those cases, when the support accompanies the ground deformations, the GRC is not significantly modified and the CV-CF method is appropriate. On the contrary, when a stiff support is installed close to the face, the modification of the GRC cannot be disregarded and, as emphasized by Barla (2016), the 'heavy method' may somehow become impractical since very high ground pressures are expected.

The present study focuses on tunneling using single shield TBM with a stiff lining installed just at the rear of the shield tail. Even if the lining is not installed very close to the face as in the 'heavy method' its stiffness may induce a modification of the GRC, depending on the ground conditions. In the appendix, the study is extended to tunnels excavated with a double shield TBM.

The results obtained with the various $\mathrm{CV}-\mathrm{CF}$ approaches as recalled in the previous sections are compared with those obtained with a 3D numerical model which permits to simulate the effect of the advancing tunnel face. A sensitivity analysis is performed in order to compare the performance of the different $\mathrm{CV}-\mathrm{CF}$ approaches. The choice of the values for the mechanical parameters of the ground and of the lining is carried out in an attempt to cover the large range of situations encountered within single shield TBM. A circular tunnel of diameter $D$ and excavated in a homogeneous ground is considered with isotropic initial stress state. A MohrCoulomb elasto-plastic model is used for the constitutive behavior of the ground and a linear elastic model is assumed for the lining.

\subsection{Numerical 3D reference model}

The axial symmetry of the problem allows for the use of a simple axisymmetric model. The numerical analysis is performed by using FLAC ${ }^{3 \mathrm{D}}$, (Itasca, 2005) (Fig2). In FLAD ${ }^{3 \mathrm{D}}$, the mesh is composed of 26500 hexahedral elements. Close to the tunnel walls where the stress gradients are high, zones with a size smaller than $0.03 D \times 0.03 D$ are generated. The size of the elements in those areas has been chosen after carrying out a sensitivity analysis. The lining is discretized into six hexahedral zones along its thickness. The tunnel excavation is modelled by incrementally removing the ground material and installing the support at a given distance from the tunnel face.

\section{Fig2. Grid geometry. Axisymmetric model (FLAC $\left.{ }^{3 \mathrm{D}}\right)$}

The sequential excavation is governed by two parameters, the step round length $s$ and the unsupported span $x_{f}$, Fig3. The step round length needs to be small enough in order to simulate a continuous excavation. Vlachopoulos and Diederichs (2009) have shown that a step of excavation below $0.4 D$ is sufficient to simulate the continuous excavation of an unsupported tunnel. In the present work, the chosen value for $s$ is $0.08 D$. The distance of support/lining installation $d$ can be expressed as:

$$
d=x_{f}+\frac{s}{2}
$$

Fig3. Sequence of calculation in the step-by-step method. 1. Installation of the lining. 2. Excavation and calculation 


\subsection{Plane-strain reference approaches}

The studied plane-strain methods are summarized in Tab2. The Classical method and the implicit method of Nguyen-Minh \& Guo are studied in combination with the expressions of the LDP of Panet (1995) and Vlachopulous and Diederichs (2009). The comparison with the method of Bernaud and Rousset is also carried out.

Tab2. Plane-strain approach: Combination of different LDP curves for various CV-CF methods for the comparison with 3D numerical results

\subsection{Sensitivity analysis}

Dimensionless variables and parameters are used in order to perform a sensitivity analysis. They are noted with the superscript (.) ${ }^{*}$.

$$
\begin{gathered}
\sigma_{\max }{ }^{*}=\frac{\sigma_{\max }}{\sigma_{0}} \\
\bar{u}(\infty)^{*}=\frac{\bar{u}(\infty) 2 G}{\sigma_{0} R} \\
d^{*}=\frac{d}{2 R} \\
R^{*}=\frac{R}{e} \\
E^{*}=\frac{E}{E_{l}}
\end{gathered}
$$

The validation of the normalization is shown in Fig4. The equilibrium points obtained in the various numerical simulations fall into a single curve once the normalization is applied. The range of ground and lining properties is summarized in Tab3.

Fig4. Results of the numerical calculations regarding the normalization. a) $\sigma_{\max }{ }^{*}$ and $\bar{u}(\infty)^{*}$ as a function of $E^{*}$, b) $\sigma_{\max }{ }^{*}$ and $\bar{u}(\infty)^{*}$ as a function of $N$ and c) $\sigma_{\max }{ }^{*}$ and $\bar{u}(\infty)^{*}$ as a function of $R^{*}$.

\section{Tab3. Range of values for the parameters}

The Poisson's ratio of the ground is kept constant and equal to $\mathrm{v}=0.25$. The Poisson's ratio of the lining is also kept constant and equal to $\mathrm{v}_{l}=0.2$. Within single shield TBM excavation, the first contact between the ground and the lining takes place between one and two diameters after the advancing face. A trade-off was carried out in order to reduce the number of calculations by fixing the value of the parameter $d^{*}$ to 1 . This assumption is on the safe side for the evaluation of the stresses in the lining. 
In practice, the most common thickness for a segmental lining is $0.4 \mathrm{~m}$ and the radius length generally varies between 4 and $6 \mathrm{~m}$ for current metro, railway or road tunnels. It leads us to the choice $R^{*}=10,12.5$ and 15 . To cover the range of relative stiffness between ground and lining, the study is carried out for $E^{*}=0.05,0.25,0.5,0.75$ and 1 . The chosen values for the friction angle $\phi$ are $20^{\circ}, 25^{\circ}, 30^{\circ}$ and $35^{\circ}$. We have assumed three different dilatancy angle ranging from zero dilatancy to associate plasticity depending on the friction angle: $\psi=0^{\circ}, \phi / 3$ and $\phi$. Finally, the calculations are performed for some representative values of $\mathrm{N}: 1,2$ and 5.

\subsection{Results and discussion}

The equilibrium states obtained from 540 axisymmetric simulations resulting from the combination of the different parameters are compared with the CV-CF approaches considered herein. In order to provide a large sensitivity analysis, parameters $N$ and $E^{*}$ have been varied independently. Note that, in practice, the ratio $E / \sigma_{c}$ varies between 200 and 1000 . Nevertheless, for values of $N$ between 1 and 5, realistic values of $E^{*}$ are covered in the proposed study.

In the present study, the maximal hoop stress obtained from the axisymmetric numerical models is compared with the one obtained from the plane-strain closed-form solutions. Fig5., Fig6. and Fig7. show the comparison between the CV-CF approaches and the results of the axisymmetric simulations in terms of $\sigma_{\max }{ }^{*}$ for a representative set of values. The total set of results is given in online resource 1 (De la Fuente et al., 2018a).

Fig5. Comparison of $\sigma_{\max }{ }^{*}$ between the different approaches when $R^{*}=12.5$ and $\phi=25^{\circ}$ for incompressible plasticity $(\psi=0)$. Classical methods on the left column and implicit methods on the right column

Fig6. Comparison of $\sigma_{\max }{ }^{*}$ between the different approaches when $R^{*}=12.5$ and $\phi=25^{\circ}$ for non-associate plasticity $(\psi=\phi / 3)$. Classical methods on the left column and implicit methods on the right column

Fig7. Comparison of $\sigma_{\max }{ }^{*}$ between the different approaches when $R^{*}=12.5$ and $\phi=25^{\circ}$ for associate plasticity $(\psi=\phi)$. Classical methods on the left column and implicit methods on the right column

It can be inferred from the study that the classical approach in combination with any LDP tends to underestimate the stress state in the lining. When a value of $N$ varying from 1 to 5 is adopted, a good agreement between the numerical simulations and the implicit method of Nguyen-Minh \& Guo combined with any LDP is observed. The implicit method of Bernaud \& Rousset shows also similar results. However, when the ground is rather soft $\left(E^{*}<0.25\right)$, due attention should be paid even though values assigned to $E^{*}$ fall within the applicability domain given by the authors. In this case, the implicit methods cannot be accurately applied as the GRC is significantly modified and the implicit methods do not take this modification into account. Finally, errors tend to increase with increasing dilatancy angle.

Regarding $\bar{u}(\infty)^{*}$ a good agreement is observed between the numerical simulations and the solution of Nguyen-Minh \& Guo when it is combined with the LDP of Panet if $N$ varies from 1 to 5, Fig8. However, for associate plasticity and $N=5$ Nguyen-Minh \& Guo method combined with the LDP of Panet underestimates $\bar{u}(\infty)^{*}$, Fig9. This is also the case for the implicit method of Bernaud and Rousset. The solution of Nguyen-Minh \& Guo combined with the LDP of Vlachopoulos \& Diederichs provides a good result for any value of $N$ and $E^{*}$ and gives the best estimate of $\bar{u}(\infty)^{*}$, (Fig8 and Fig9). Note that errors tend to increase with increasing dilatancy angle. The total set of results for $\bar{u}(\infty)^{*}$ is given in online resource 2 (De la Fuente et al., 2018b). 
Fig8. Comparison of $\bar{u}(\infty)^{*}$ between the different approaches when $R^{*}=12.5$ and $\phi=25^{\circ}$ for incompressible plasticity $(\psi=0)$. Classical methods on the left column and implicit methods on the right column

Fig9. Comparison of $\bar{u}(\infty)^{*}$ between the different approaches when $R^{*}=12.5$ and $\phi=25^{\circ}$ for associate plasticity $(\psi=\phi)$. Classical methods on the left column and implicit methods on the right column

The effect of the dilatancy of the ground is further highlighted by considering an unlined tunnel. The difference between the axisymmetric numerical computations (.)axy and the planestrain analytical results $(.)_{p s}$ are plotted in Fig. 10 and 11 in terms of $\left(\left(u(\infty)^{*}{ }_{\text {axi }}-\right.\right.$ $\left.u(\infty)_{p s}^{*}\right) / u(\infty)_{p s}^{*}$ ). When the flow rule is strongly non-associated (values of $\psi$ between 0 and $\phi / 3)$, which is a case commonly encountered in practice, both approaches give very similar results for the final radial displacement. However, when assuming an associated flow rule, the discrepancy between the results is amplified for higher values of $N$ and can reach almost $20 \%$ (Fig10). Fig11 shows that even for $N=5$, the results are only slightly affected by the friction angle which confirms that the dilatancy angle is the controlling parameter.

Fig10. Effect of $N$ on the difference between the numerical simulation and the plane strain closed form solution for the radial displacement at the tunnel wall $\left(\phi=35^{\circ}\right.$ and $\left.\mathrm{v}=0.25\right)$

Fig11. Effect of the friction angle $\phi$ on the difference between the numerical simulation and the plane strain closed form solution for the radial displacement at the tunnel wall $(N=10$ and $\mathrm{u}=0.25)$

\section{Empirical formulas for preliminary design in the case of single shield TBM}

In the previous section we have discussed the relevance of the implicit methods for reliable predictions of the lining stress state at equilibrium. These methods are based on a modification of the radial displacement of the tunnel wall at the instant of installation of the lining. The CV$\mathrm{CF}$ method is then applied directly. Another approach is to make use of the large number of the computations performed in the first part of the study in order to propose empirical relationships which directly give the equilibrium state. Such empirical formula can be very useful for preliminary design. In the present work, we provide a set of empirical expressions covering a large range of rock and support conditions. Therefore, three different mathematical formulas are proposed. The choice of the appropriate expression for each configuration is based on the value of a dimensionless parameter $F$ which depends on the mechanical parameters and is given in equation 41 where the friction and dilatancy angles $\phi$ and $\psi$ are expressed in degrees. In order to be consistent with the configuration of a single shield TBM, we assume $d^{*}=1$.

$$
F=0.922+0.0224 R^{*}+N\left(\frac{3.88}{\phi}+9.66 * 10^{-4} *(\psi+1)-0.063\right)+0.365 \frac{E^{*}}{N}-0.76 \log _{10}\left(100 E^{*}\right)
$$

For $F<0.4, \sigma_{\text {max }}{ }^{*}$ is given by equation 42 .

$$
\begin{gathered}
\sigma_{\text {max }}{ }^{*}=0.42+0.004 \phi+R^{*}\left(0.0082-0.0096 \frac{E^{*}}{N}\right)-N\left(0.123+\frac{1}{\phi}\left(0.0685 N+\frac{64.57}{\phi}-7.79\right)-0.000174(\psi+1)\right) \\
+E^{*}\left(0.0027 \frac{1}{E^{* 3}}+0.1954 \frac{1}{N}+\frac{(\psi+1)}{\phi}\left(-\frac{0.1}{N}+0.0916\right)\right)-0.3455 \log _{10}\left(100 E^{*}\right)
\end{gathered}
$$

For $0.4 \leq F<0.8, \sigma_{\max }{ }^{*}$ is given by equation 43 : 


$$
\begin{aligned}
\sigma_{\text {max }}^{*}=1.1149+ & 0.0227 R^{*}+\psi(0.0038-0.0001 \psi)+0.04 \frac{1}{(\psi+1)^{2}} \\
& -N\left(0.0879+\frac{0.00826}{E^{*}}-0.000148 \frac{N}{E^{* 2}}+0.158 \frac{N}{\phi}+\frac{41.785}{\phi^{2}}+\frac{4.06}{E^{*} \cdot \phi^{2}}-0.000463(\psi+1)-\frac{8.3}{\phi}\right) \\
+ & E^{*}\left(-\frac{0.253}{N . \phi}+\frac{0.244}{\phi}\right)(\psi+1)-0.96 \log _{10}\left(100 E^{*}\right)
\end{aligned}
$$

Finally, if $F \geq 0.8$,

$$
\begin{aligned}
\sigma_{\text {max }}{ }^{*}=0.9617- & 0.0143 \phi+0.0458 R^{*}-194.85 \frac{1}{\phi^{2}}+0.0647 \frac{1}{(\psi+1)^{2}} \\
& +N\left(-0.06 \frac{N}{\phi}+\frac{69.55}{\phi^{2}}-0.0000357(\psi+1)^{2}+0.00192(\psi+1)+\frac{0.095}{E^{*} . \phi}-\frac{1.303}{E^{*} \cdot \phi^{2}}\right) \\
& +E^{*}\left(-0.202 E^{*}+0.000267 \frac{1}{E^{* 3}}+0.478 \frac{(\psi+1)}{\phi}\right)-0.675 \log _{10}\left(100 E^{*}\right)
\end{aligned}
$$

Following the same procedure, a unique expression is proposed for $\bar{u}(\infty)^{*}$ :

$$
\begin{aligned}
\bar{u}(\infty)^{*}=1.6244+ & 0.012 R^{*}+\phi\left(1.3 \cdot 10^{-5} \phi^{2}-\frac{0.027}{N}\right) \\
& +N\left(0.0178 E^{*}+0.01855(\psi+1)+\frac{0.543}{\psi+1}-0.017 \phi+\frac{5}{\phi}-\frac{21.99}{\phi(\psi+1)}+\frac{4.076 N}{\phi(\psi+1)}-\frac{0.24 N^{2}}{\phi(\psi+1)}\right) \\
+ & \frac{(\psi+1)}{\phi}\left(-0.0146 N^{3}+0.323 N^{2}-0.99 N\right)
\end{aligned}
$$

As it can be seen in Tab4, by using the proposed formulation, the point of equilibrium for arbitrary combinations of the parameters within the studied range of values can be obtained with acceptable accuracy.

\section{Tab4. Validation of the empirical formulation}

\section{Conclusion}

Due attention should be paid when applying the CV-CF methods in a pre-design stage of a circular tunnel excavated in full section with a stiff support/lining system. In this work, we have compared different CV-CF methods which are based on plane-strain assumptions with a numerical model which captures the spatial effects at the vicinity of the tunnel face

It was shown that for reliable evaluation of the stress state at equilibrium in the lining, implicit methods (Nguyen-Minh \& Guo or Bernaud \& Rousset) should be used and can be combined with any LDP expression (Panet, Corbetta or Vlachopoulos \& Diederichs) for values of the stability number $N$ ranging from one to five and relatively hard rock mass $\left(E^{*}>0.25\right)$. The evaluation of the radial convergence of the ground is good with any CV-CF approach.

The influence of the ground dilatancy on the displacement around the tunnel is also highlighted for an unlined tunnel. It is shown that for low values of the dilatancy angle, the discrepancy between the 2D closed-form solution and the 3D numerical computations is small but tends to increase with increasing dilatancy angle.

Finally, a set of empirical formula are proposed that can easily provide reliable predictions of the equilibrium state for single shield TBM for a large range of ground and support/lining properties. The predicted displacements can be obtained with an accuracy of about $20 \%$ and the stresses in the lining with an accuracy of $10 \%$. 


\section{Appendix: CV-CF methods for double shield TBM}

In an attempt to assess the applicability of the CV-CF methods to tunnels excavated with a double shield TBM some numerical simulations have been carried out by fixing the value of the parameter $d^{*}$ to 2 . The results are summarized in Fig12 and Fig13. It is inferred from the results that a classical CV-CF method in combination with the LDP of Panet provides an equilibrium state which is in agreement with the numerical simulations if $E^{*}>0.25$. The effect of the stiffness of the lining on the GRC is less than in the case of single shield tunneling. The implicit method of Guo \& Minh combined with an LDP of Panet provides also reasonable results except for $N=5$ where the implicit method reaches its limits $\left(N=5\right.$ and $\left.d^{*}=2\right)$.

Fig12. Comparison of $\sigma_{\max }{ }^{*}$ between the different approaches when $d^{*}=2, R^{*}=12.5$ and $\phi=20^{\circ}$ for incompressible plasticity $(\psi=0)$. Classical method on the left column and implicit method of Nguyen-Minh \& Guo on the right column

Fig13. Comparison of $\bar{u}(\infty)^{*}$ between the different approaches when $d^{*}=2, R^{*}=12.5$ and $\phi=25^{\circ}$ for incompressible plasticity $(\psi=0)$. Classical method on the left column and implicit method of Nguyen-Minh \& Guo on the right column

\section{References}

AFTES (2002) Recommandations pour l'emploi de la méthode convergence-confinement. Tunnels et Ouvrages Souterrains 170:79-89 (in French)

Barla G (2016) Full-face excavation of large tunnels in difficult conditions. Journal of Rock Mechanics and Geotechnical Engineering, 8(3):294-303

Bernaud D, Rousset G (1992) La «nouvelle méthode implicite » pour l'étude du dimensionnement des tunnels. Revue française de Géotechnique 60:5-26 (in French)

Bernaud D, Benamar I, Rousset G (1994) La «nouvelle méthode implicite » pour le calcul des tunnels dans les milieux élastoplastiques et viscoplastiques. Revue française de Géotechnique, 68:3-19 (in French)

Cantieni L, Anagnostou G (2009) The effect of the stress path on squeezing behavior in tunneling. Rock mechanics and rock engineering 42(2):289-318

Corbetta F, Bernaud D, Nguyen-Minh D (1991) Contribution à la méthode convergenceconfinement par le principe de la similitude. Revue française de géotechnique, 54:5-11 (in French)

De Labriolle GC (2017) Amélioration des méthodes analytiques basées sur des concepts, simples pour le dimensionnement des tunnels superficiels et profonds en sol meuble. Revue Française de Géotechnique 151:2 (in French)

De la Fuente M, Taherzadeh R, Sulem J, Nguyen XS, Subrin D (2018a) Maximal hoop stress developed in the lining of a tunnel excavated with a single shield TBM at the state of equilibrium (comparison between different calculation methods), on line resource 1 (version 2), Zenodo, DOI: https://doi.org/10.5281/zenodo.3960646

De la Fuente M, Taherzadeh R, Sulem J, Nguyen XS, Subrin D (2018b) Radial displacement at the tunnel wall of a tunnel excavated with a single shield TBM at the state of equilibrium (comparison between different calculation methods), on line resource 2 (version 2), Zenodo, DOI: https://doi.org/10.5281/zenodo.3960652

Eisenstein, Z, Branco P (1991) Convergence-Confinement method in shallow tunnels. Tunnelling and underground space technology, 6(3):343-346

Flügge W (1960) Stresses in shells. New York

Gonzalez-Nicieza C, Alvarez-Vigil AE, Menendez-Diaz A, Gonzalez-Palacio C (2008) 
Influence of the depth and shape of a tunnel in the application of the convergenceconfinement method. Tunnelling and Underground Space Technology 21(1):25-37

Itasca (2005) FLAC3D Numerical Software Package. Itasca Consulting Group Inc, Lagrangian Analysis of Continua

Lee SW, Jung JW, Nam SW, Lee IM (2007). The influence of seepage forces on ground reaction curve of circular opening. Tunnelling and Underground Space Technology, 22(1):28-38.

Nguyen-Minh D, Guo C (1996) Recent progress in convergence-confinement method. Eurock'96, Barla (ed.), Turin 855-860

Oreste, P (2009) The Convergence-Confinement Method: Roles and Limits in Modern Geomechanical Tunnel Design. American Journal of Applied Sciences 6(4) :757-771

Panet M, Guellec P (1974) Contribution à l'étude du soutènement d'un tunnel à l'arrière du front de taille. Progrès en mécanique des roches comptes rendus du 3eme congrès de la société internationale de mécanique des roches, Denver (2) (in French)

Panet M, Guenot A (1983) Analysis of convergence behind the face of a tunnel. Tunnelling 82, proceeding of the 3rd international symposium, Brighton 197-204

Panet M (1995) Le calcul des tunnels par la méthode convergence-confinement, Presses de l'Ecole Nationale des ponts et chaussées, Paris (in French)

Ramoni M, Lavdas N, Anagnostou G (2011) Squeezing loading of segmental linings and the effect of backfilling. Tunneling and Underground Space technology 26(6):692-717

Schürch R, Anagnostou, G (2012) The applicability of the ground response curve to tunnelling problems that violate rotational symmetry. Rock Mechanics and Rock Engineering 45(1):110

Sulem, J., Panet, M., \& Guenot, A. (1987). An analytical solution for time-dependent displacements in a circular tunnel. International Journal of Rock Mechanics and Mining Sciences \& Geomechanics Abstracts, 24(3), 155-164.

Tran-Manh, H, Sulem, J, Subrin, D (2015) A Closed-Form Solution for Tunnels with Arbitrary Cross Section Excavated in Elastic Anisotropic Ground. Rock Mechanics and Rock Engineering, 48(1):277-288

Vlachopoulos N, Diederichs MS (2009) Improved longitudinal displacement profiles for convergence confinement analysis of deep tunnels. Rock Mechanics and Rock Engineering 42(2):131-146

Vlachopoulos N, Diederichs MS (2014) Appropriate uses and practical limitations of 2D numerical analysis of tunnels and tunnel support response. Geotechnical and Geological Engineering 32(2):469-488

Wong H, Subrin D, Dias D (2006) Convergence confinement analysis of a bolt-supported tunnel using the homogenization method. Canadian geotechnical journal 43(5):462-483. 
Tab1. Summary for the application of the CV-CF methods

\begin{tabular}{l|c|c}
\hline \multicolumn{1}{c|}{ Method } & $\begin{array}{c}\overline{\boldsymbol{u}}(\boldsymbol{d}) \\
\text { (from equations) }\end{array}$ & $\begin{array}{c}\text { Equilibrium state } \\
\text { (by solving the system of equations) }\end{array}$ \\
\hline Classical (elastic ground) & 16 & 3 and 12 \\
Classical (elasto-plastic ground) & 21,24 or 25 & 4 and 12 \\
\hline Bernaud \& Rousset (elastic ground) & 30 & 3,12 and 30 \\
Bernaud \& Rousset (elasto-plastic ground) & 30 & 4,12 and 30 \\
\hline Guo \& Minh (elastic ground) & 33 & 3,12 and 33 \\
Guo \& Minh (elasto-plastic ground) & 33 & 4,12 and 33 \\
\hline
\end{tabular}


Tab2. Plane-strain approach: Combination of different LDP curves for various CV-CF methods for the comparison with 3D numerical results

\begin{tabular}{c} 
Classical CV-CF Method - LDP Panet (1995) \\
$\frac{\text { Classical CV-CF Method - LDP Vlachopoulos and Diederichs (V \& D) (2009) }}{\text { Guo and Minh Method (M \& G) - LDP Panet (1995) }}$ \\
Guo and Minh Method (M \& G) - LDP Vlachopoulos and Diederichs (V \& D) (2009) \\
\hline Bernaud and Rousset Method (B \& R) - LDP Panet and Guénot (P \& G) (1983)
\end{tabular}


Tab3. Range of values for the parameters

\begin{tabular}{c|c}
\hline Parameter & Values \\
\hline $\mathrm{u}$ & 0.25 \\
$\mathrm{v}_{l}$ & 0.2 \\
$d^{*}$ & 1 \\
$R^{*}$ & $10,12.5$ and 15 \\
$E^{*}$ & $0.05,0.25,0.5,0.75$ and 1 \\
$\phi$ & $20^{\circ}, 25^{\circ}, 30^{\circ}$ and $35^{\circ}$ \\
$\psi$ & $0^{\circ}, \phi / 3$ and $\phi$ \\
$N$ & 1,2 and 5 \\
\hline
\end{tabular}


Tab4. Validation of the empirical formulation

\begin{tabular}{|c|c|c|c|c|c|c|c|c|c|c|c|c|}
\hline$d^{*}$ & $R^{*}$ & $E^{*}$ & $N$ & $\phi\left(^{\circ}\right)$ & $\psi\left({ }^{\circ}\right)$ & $F$ & $\sigma_{(F L A C 3 D)}{ }^{*}$ & 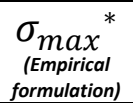 & $\begin{array}{c}\text { Error } \\
\text { (\%) }\end{array}$ & $\underset{\left(F^{\prime} A C^{30}\right)}{\bar{u}}$ & 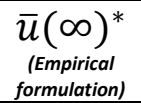 & $\begin{array}{c}\text { Error } \\
(\%)\end{array}$ \\
\hline 1 & 10 & 0.05 & 2 & 20 & 6.7 & 0.90 & 0.736 & 0.712 & -3.3 & 1.165 & 1.325 & 13.7 \\
\hline 1 & 10 & 0.05 & 2.5 & 20 & 6.7 & 0.97 & 0.826 & 0.813 & -1.5 & 1.378 & 1.463 & 6.2 \\
\hline 1 & 10 & 0.05 & 3 & 20 & 6.7 & 1.04 & 0.910 & 0.913 & 0.4 & 1.619 & 1.632 & 0.8 \\
\hline 1 & 10 & 0.05 & 3.5 & 20 & 6.7 & 1.10 & 0.996 & 1.012 & 1.6 & 1.871 & 1.833 & -2.0 \\
\hline 1 & 10 & 0.05 & 4 & 20 & 6.7 & 1.17 & 1.091 & 1.109 & 1.7 & 2.134 & 2.066 & -3.2 \\
\hline 1 & 10 & 0.05 & 4.5 & 20 & 6.7 & 1.24 & 1.178 & 1.204 & 2.2 & 2.400 & 2.326 & -3.1 \\
\hline 1 & 10 & 0.05 & 1.4 & 20 & 6.7 & 0.83 & 0.651 & 0.594 & -8.7 & 0.985 & 1.186 & 20.4 \\
\hline 1 & 10 & 0.05 & 2 & 20 & 6.7 & 0.90 & 0.736 & 0.712 & -3.3 & 1.165 & 1.325 & 13.7 \\
\hline 1 & 10 & 0.05 & 2.6 & 20 & 6.7 & 0.98 & 0.841 & 0.828 & -1.6 & 1.409 & 1.485 & 5.4 \\
\hline 1 & 10 & 0.05 & 3.1 & 20 & 6.7 & 1.06 & 0.930 & 0.942 & 1.3 & 1.686 & 1.686 & 0.0 \\
\hline 1 & 10 & 0.05 & 3.7 & 20 & 6.7 & 1.13 & 1.033 & 1.054 & 2.0 & 1.983 & 1.929 & -2.7 \\
\hline 1 & 10 & 0.05 & 4.3 & 20 & 6.7 & 1.21 & 1.136 & 1.163 & 2.4 & 2.288 & 2.211 & -3.3 \\
\hline 1 & 10 & 0.05 & 1.6 & 20 & 6.7 & 0.85 & 0.675 & 0.630 & -6.7 & 1.029 & 1.228 & 19.3 \\
\hline 1 & 10 & 0.05 & 2 & 20 & 6.7 & 0.90 & 0.734 & 0.712 & -3.0 & 1.165 & 1.325 & 13.7 \\
\hline 1 & 10 & 0.05 & 2.4 & 20 & 6.7 & 0.95 & 0.804 & 0.793 & -1.3 & 1.333 & 1.433 & 7.5 \\
\hline 1 & 10 & 0.05 & 2.8 & 20 & 6.7 & 1.01 & 0.876 & 0.874 & -0.3 & 1.520 & 1.560 & 2.7 \\
\hline 1 & 10 & 0.05 & 3.2 & 20 & 6.7 & 1.06 & 0.948 & 0.953 & 0.6 & 1.713 & 1.708 & -0.3 \\
\hline 1 & 10 & 0.05 & 3.6 & 20 & 6.7 & 1.12 & 1.009 & 1.031 & 2.2 & 1.921 & 1.877 & -2.3 \\
\hline 1 & 10 & 0.13 & 2 & 20 & 6.7 & 0.59 & 0.484 & 0.467 & -3.4 & 1.192 & 1.328 & 11.4 \\
\hline 1 & 10 & 0.20 & 2 & 20 & 6.7 & 0.47 & 0.387 & 0.375 & -3.2 & 1.205 & 1.330 & 10.4 \\
\hline 1 & 10 & 0.27 & 2 & 20 & 6.7 & 0.39 & 0.335 & 0.344 & 2.6 & 1.216 & 1.332 & 9.6 \\
\hline 1 & 10 & 0.33 & 2 & 20 & 6.7 & 0.33 & 0.302 & 0.301 & -0.2 & 1.226 & 1.335 & 8.9 \\
\hline 1 & 10 & 0.40 & 2 & 20 & 6.7 & 0.28 & 0.274 & 0.271 & -1.1 & 1.234 & 1.337 & 8.4 \\
\hline 1 & 10 & 0.35 & 2 & 20 & 6.7 & 0.31 & 0.293 & 0.293 & -0.2 & 1.228 & 1.335 & 8.8 \\
\hline 1 & 10 & 0.55 & 2 & 20 & 6.7 & 0.20 & 0.227 & 0.225 & -0.8 & 1.247 & 1.343 & 7.6 \\
\hline 1 & 10 & 0.75 & 2 & 20 & 6.7 & 0.13 & 0.186 & 0.187 & 0.6 & 1.260 & 1.350 & 7.1 \\
\hline 1 & 10 & 0.95 & 2 & 20 & 6.7 & 0.09 & 0.157 & 0.163 & 4.2 & 1.268 & 1.357 & 7.0 \\
\hline 1 & 10 & 0.17 & 2 & 20 & 6.7 & 0.52 & 0.427 & 0.419 & -1.8 & 1.199 & 1.329 & 10.9 \\
\hline 1 & 10 & 0.30 & 2 & 20 & 6.7 & 0.35 & 0.316 & 0.321 & 1.4 & 1.221 & 1.334 & 9.2 \\
\hline 1 & 10 & 0.43 & 2 & 20 & 6.7 & 0.26 & 0.262 & 0.258 & -1.2 & 1.237 & 1.338 & 8.2 \\
\hline 1 & 10 & 0.57 & 2 & 20 & 6.7 & 0.19 & 0.223 & 0.221 & -1.1 & 1.249 & 1.343 & 7.6 \\
\hline 1 & 10 & 0.70 & 2 & 20 & 6.7 & 0.15 & 0.194 & 0.195 & 0.5 & 1.257 & 1.348 & 7.2 \\
\hline 1 & 11.43 & 0.05 & 2 & 20 & 6.7 & 0.93 & 0.795 & 0.777 & -2.2 & 1.128 & 1.342 & 18.9 \\
\hline 1 & 14.29 & 0.05 & 2 & 20 & 6.7 & 1.00 & 0.830 & 0.908 & 9.5 & 1.134 & 1.376 & 21.3 \\
\hline 1 & 12 & 0.05 & 2 & 20 & 6.7 & 0.95 & 0.812 & 0.804 & -1.0 & 1.130 & 1.349 & 19.4 \\
\hline
\end{tabular}




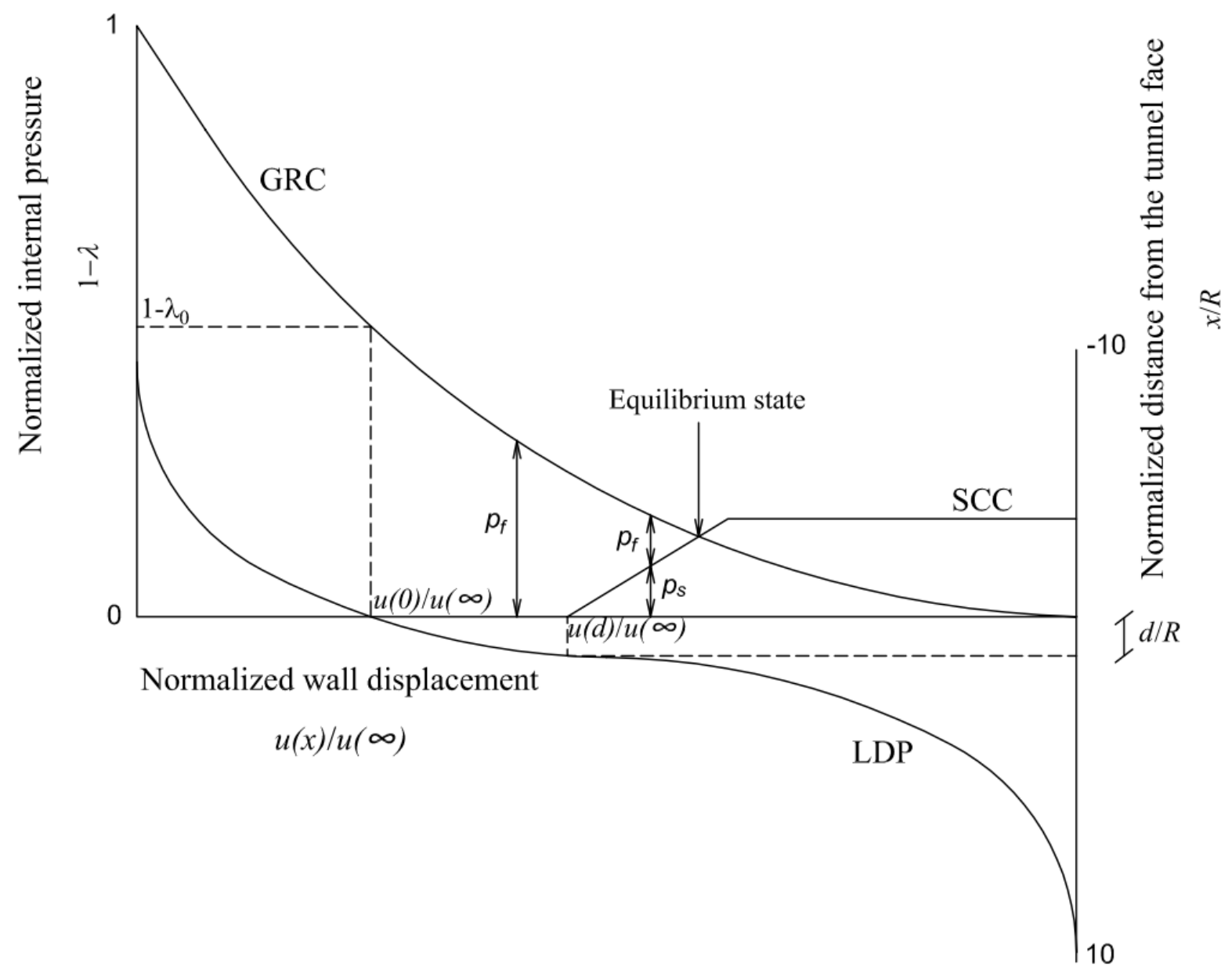

Fig1. Schematic representation of the curves employed in the CV-CF method (Designed with AutoCAD) 


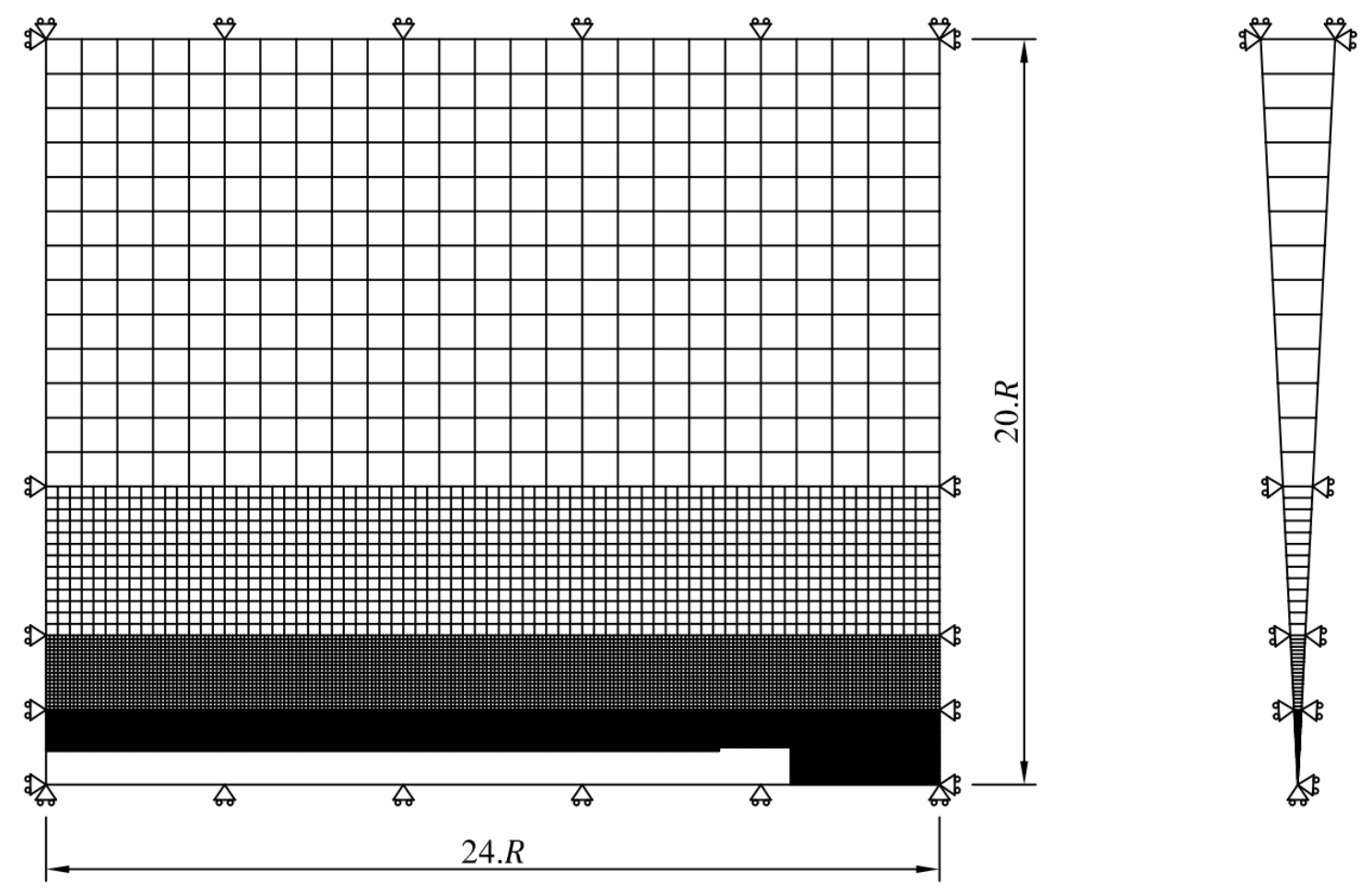

Fig2. Grid geometry. Axisymmetric model (FLAC ${ }^{3 \mathrm{D}}$ ) (Designed with AutoCAD) 


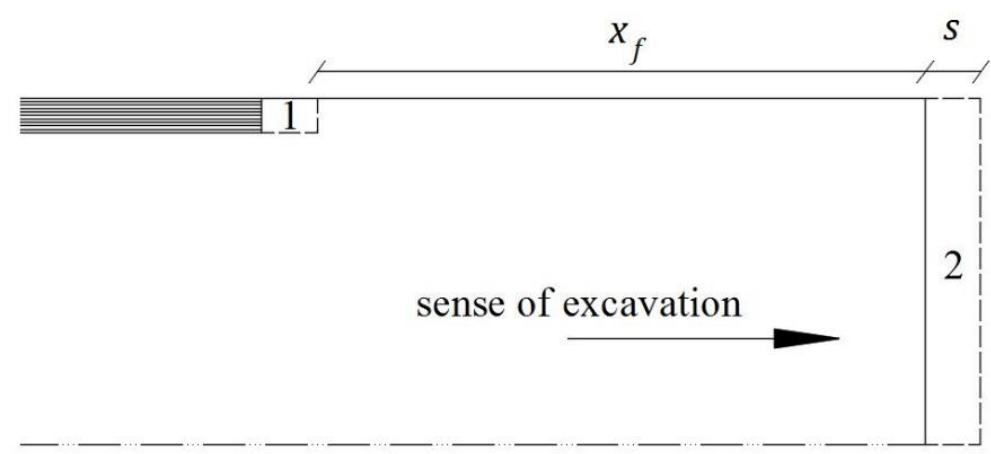

Fig3. Sequence of calculation in the step-by-step method. 1. Installation of the lining. 2. Excavation and calculation (Designed with AutoCAD) 

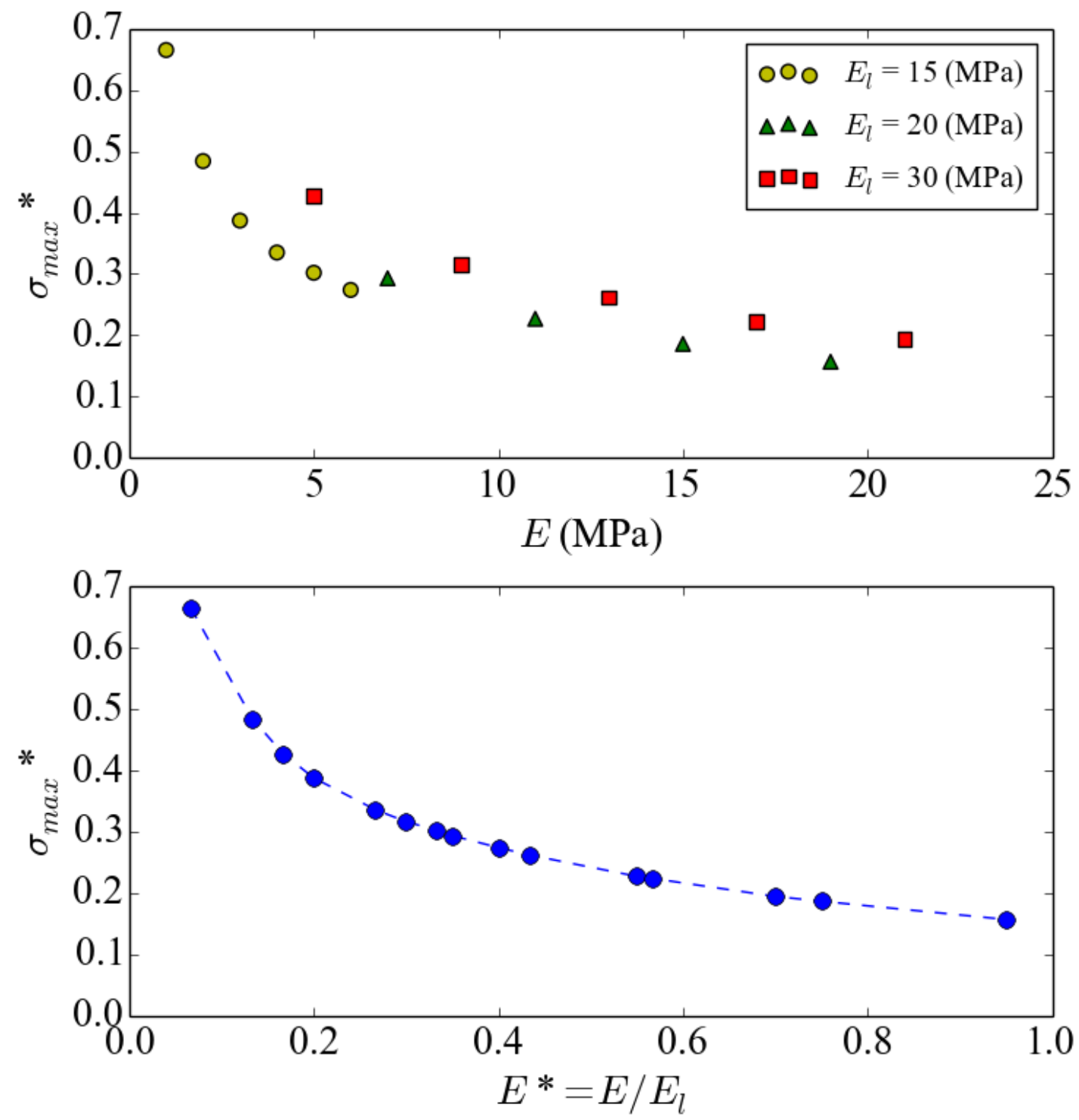

a) 

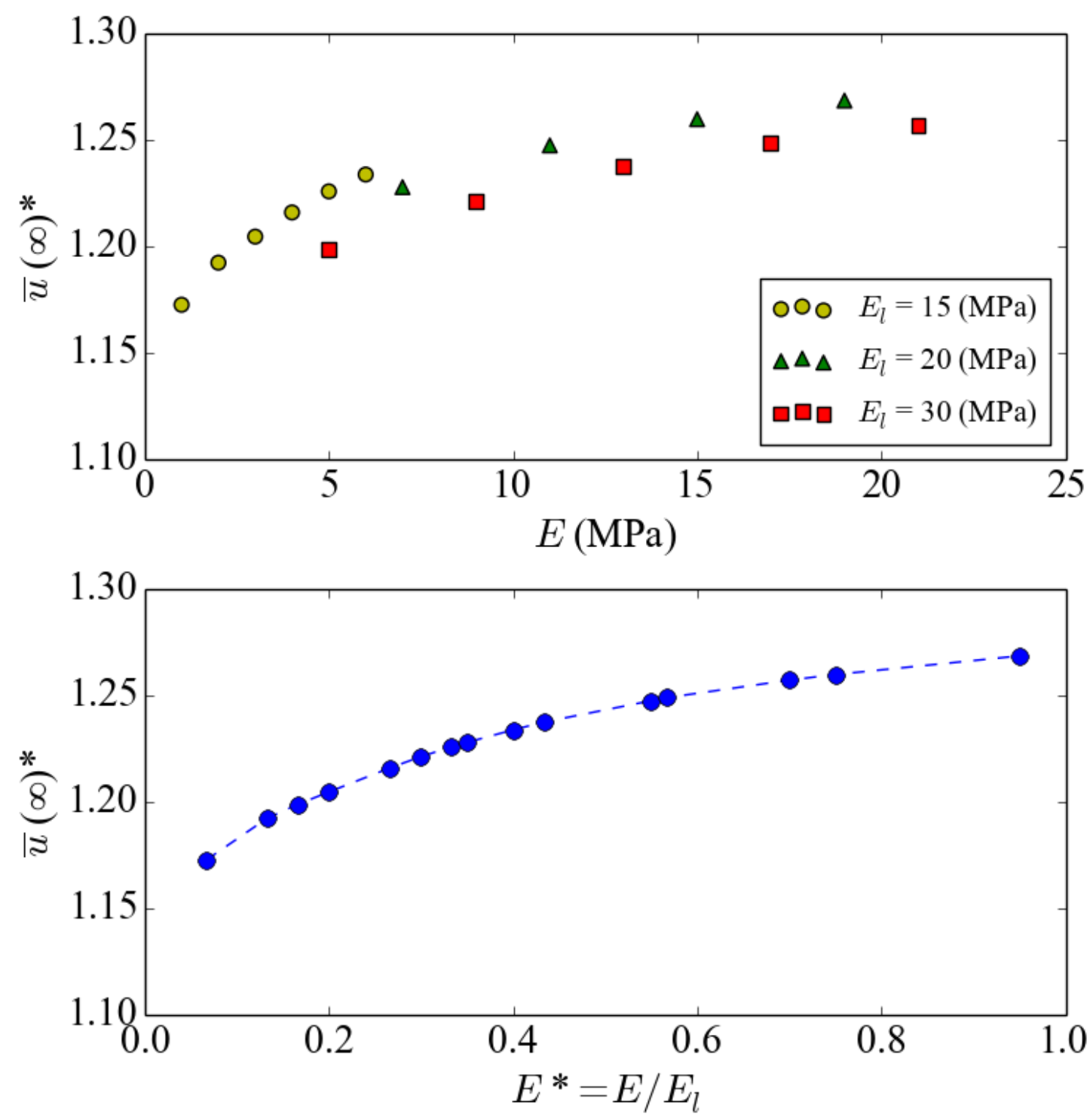

a) 

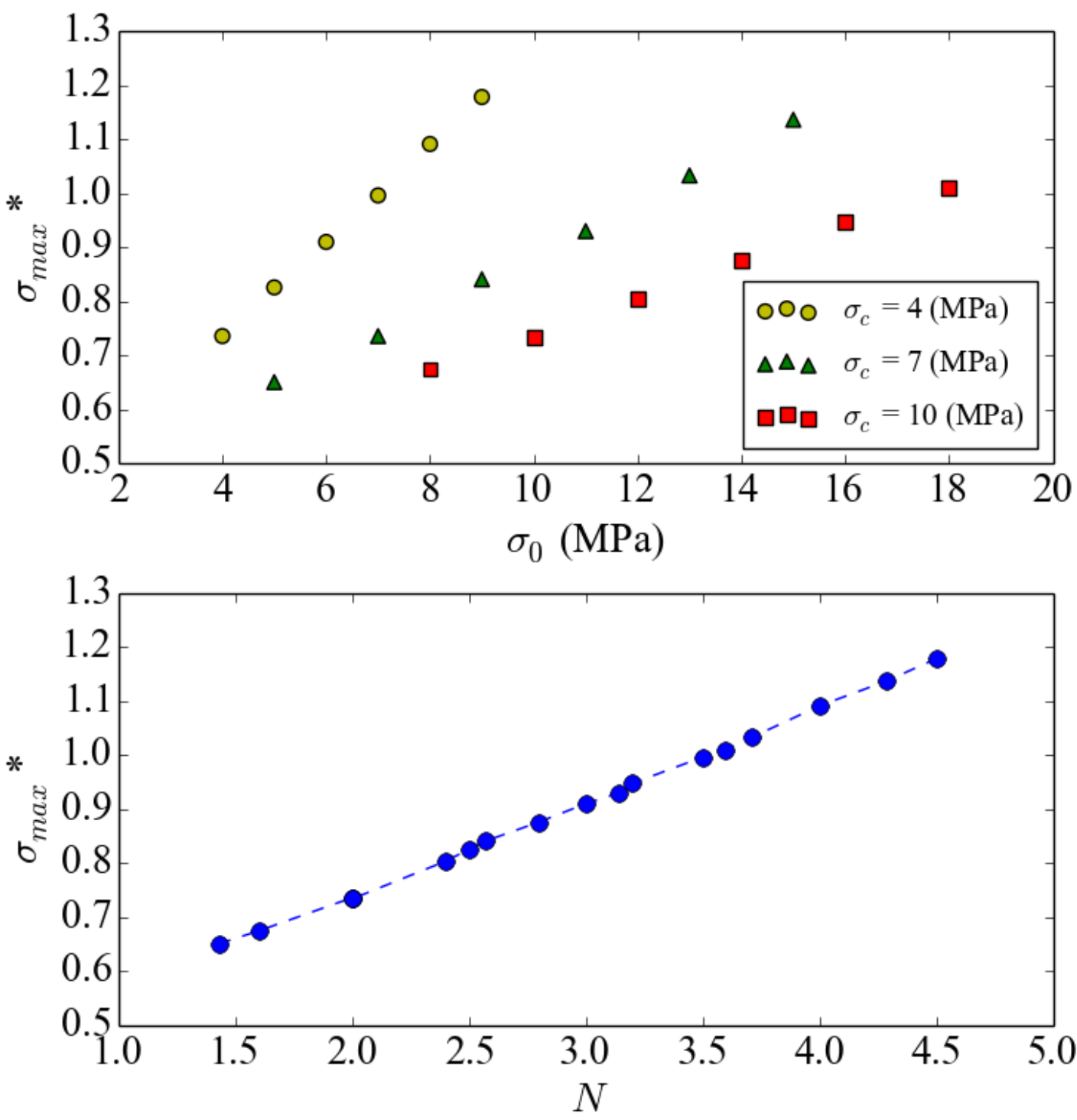

b) 

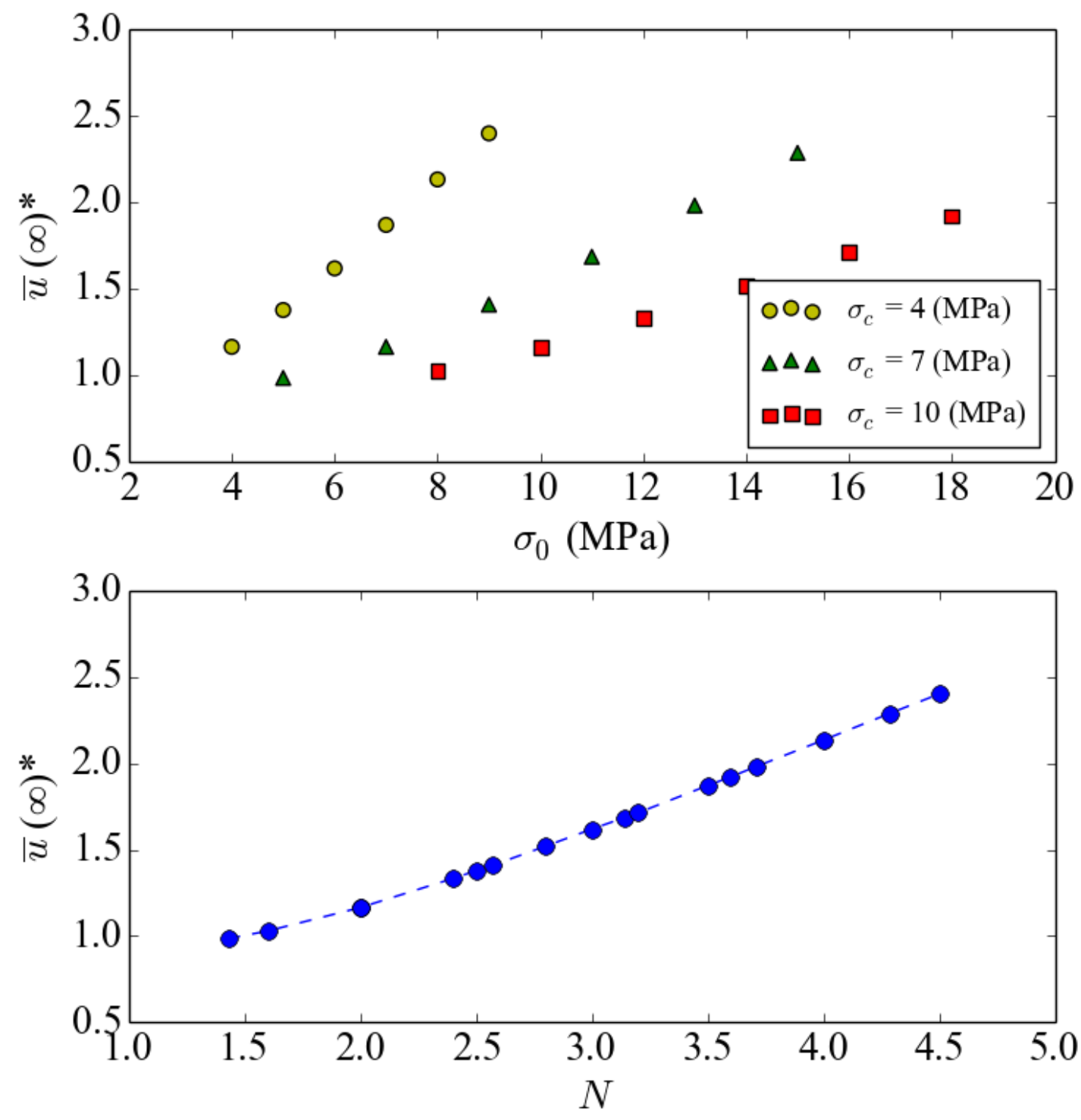

b) 

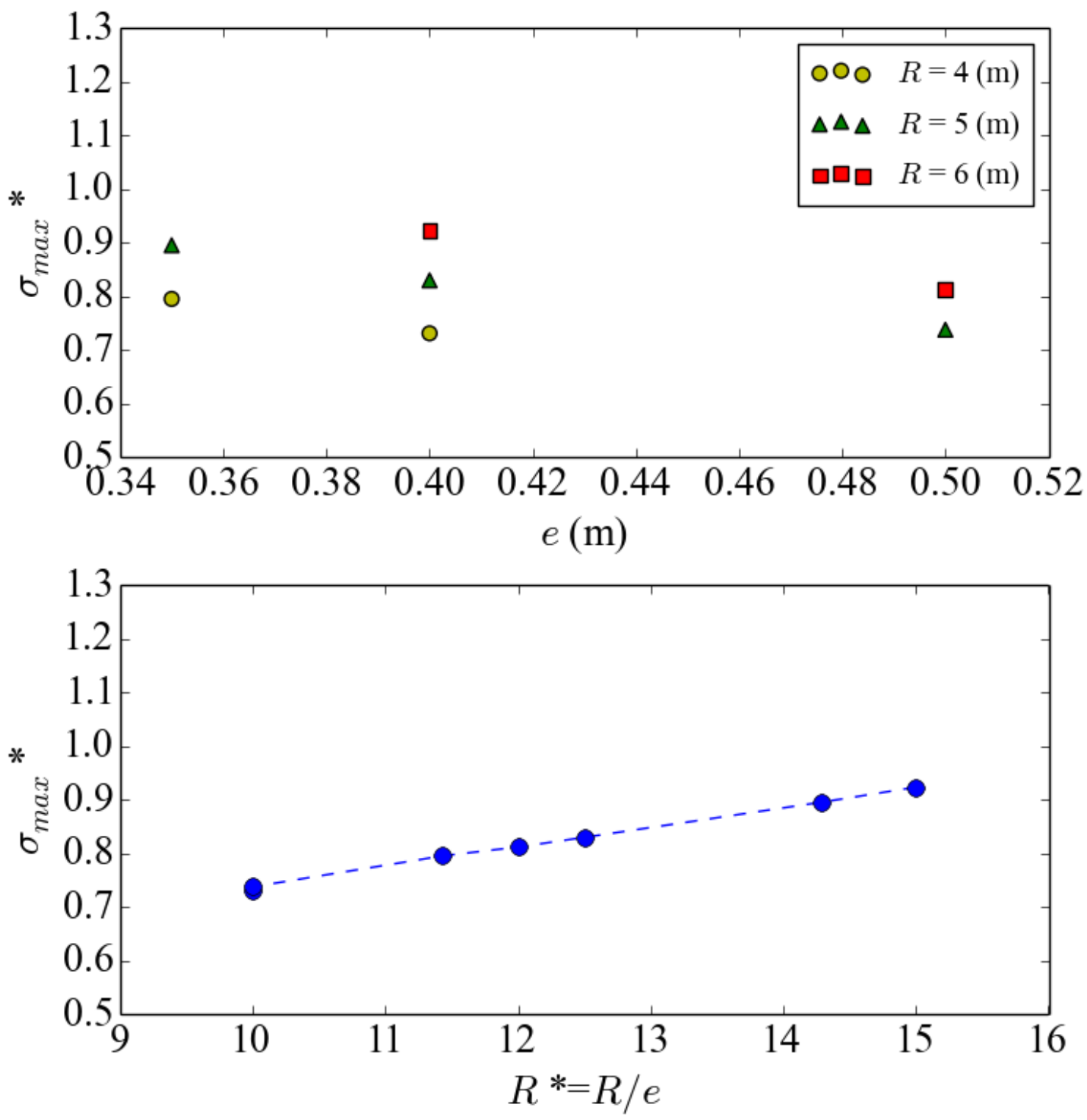

c) 

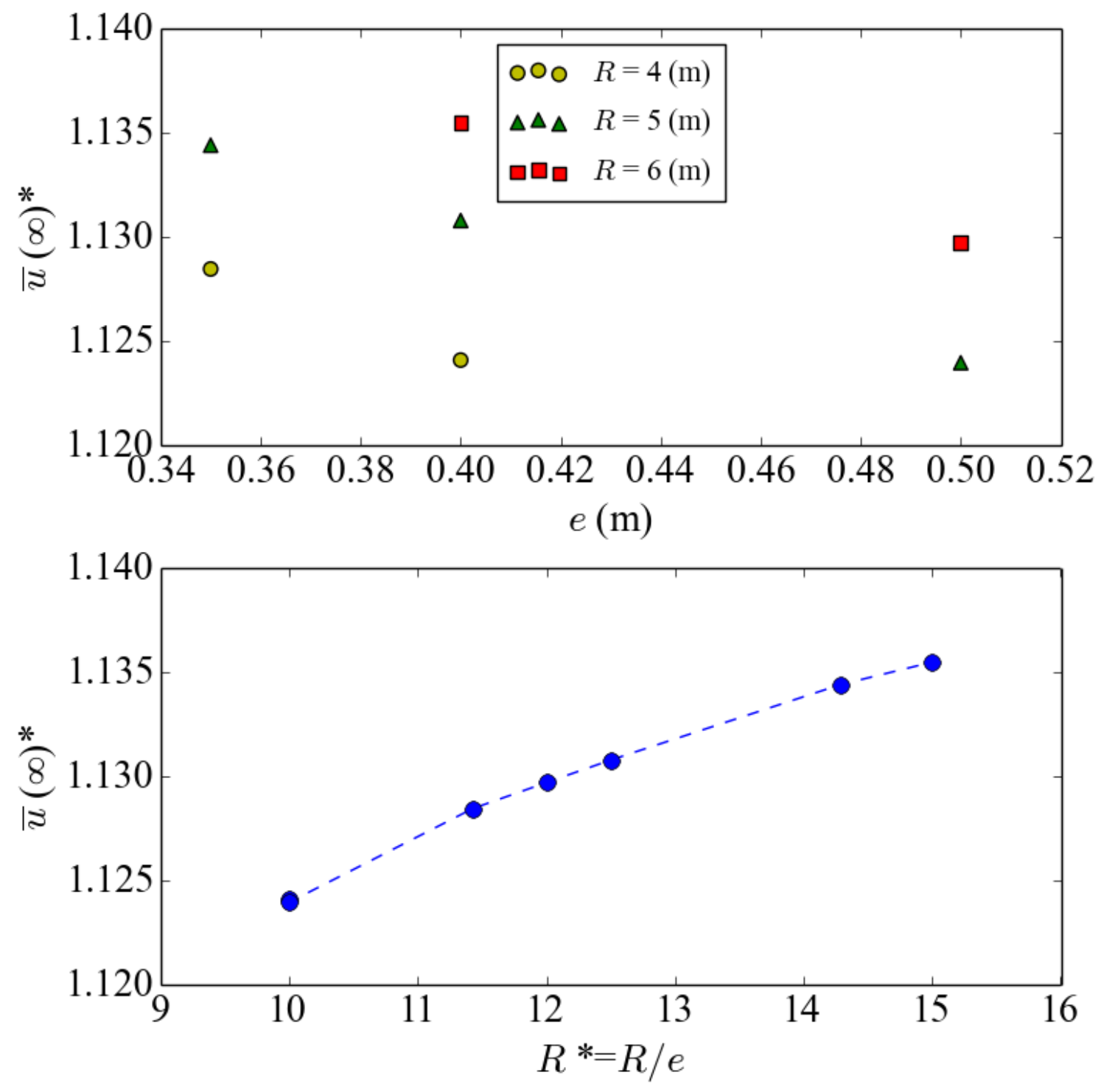

c)

Fig4. Results of the numerical calculations regarding the normalization. a) $\sigma_{\max }{ }^{*}$ and $\bar{u}(\infty)^{*}$ as a function of $\left.E^{*}, \mathbf{b}\right) \sigma_{\max }{ }^{*}$ and $\bar{u}(\infty)^{*}$ as a function of $\mathrm{N}$ and c) $\sigma_{\max }{ }^{*}$ and $\bar{u}(\infty)^{*}$ as a function of $R^{*}$. 

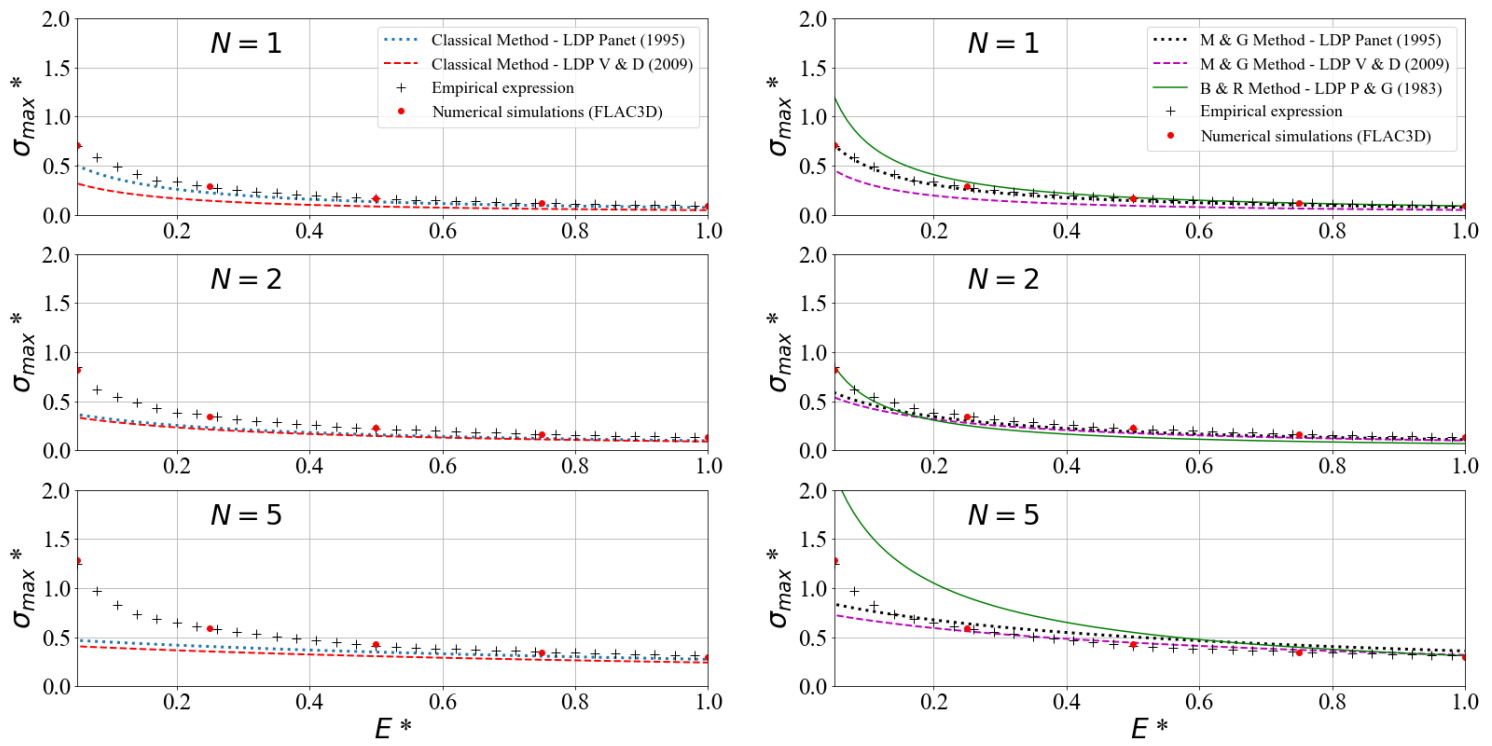

Fig5. Comparison of $\sigma_{\max }{ }^{*}$ between the different approaches when $R^{*}=12.5$ and $\phi=25^{\circ}$ if $\psi$ is equal to 0 . Classical methods on the left column and implicit methods on the right column 

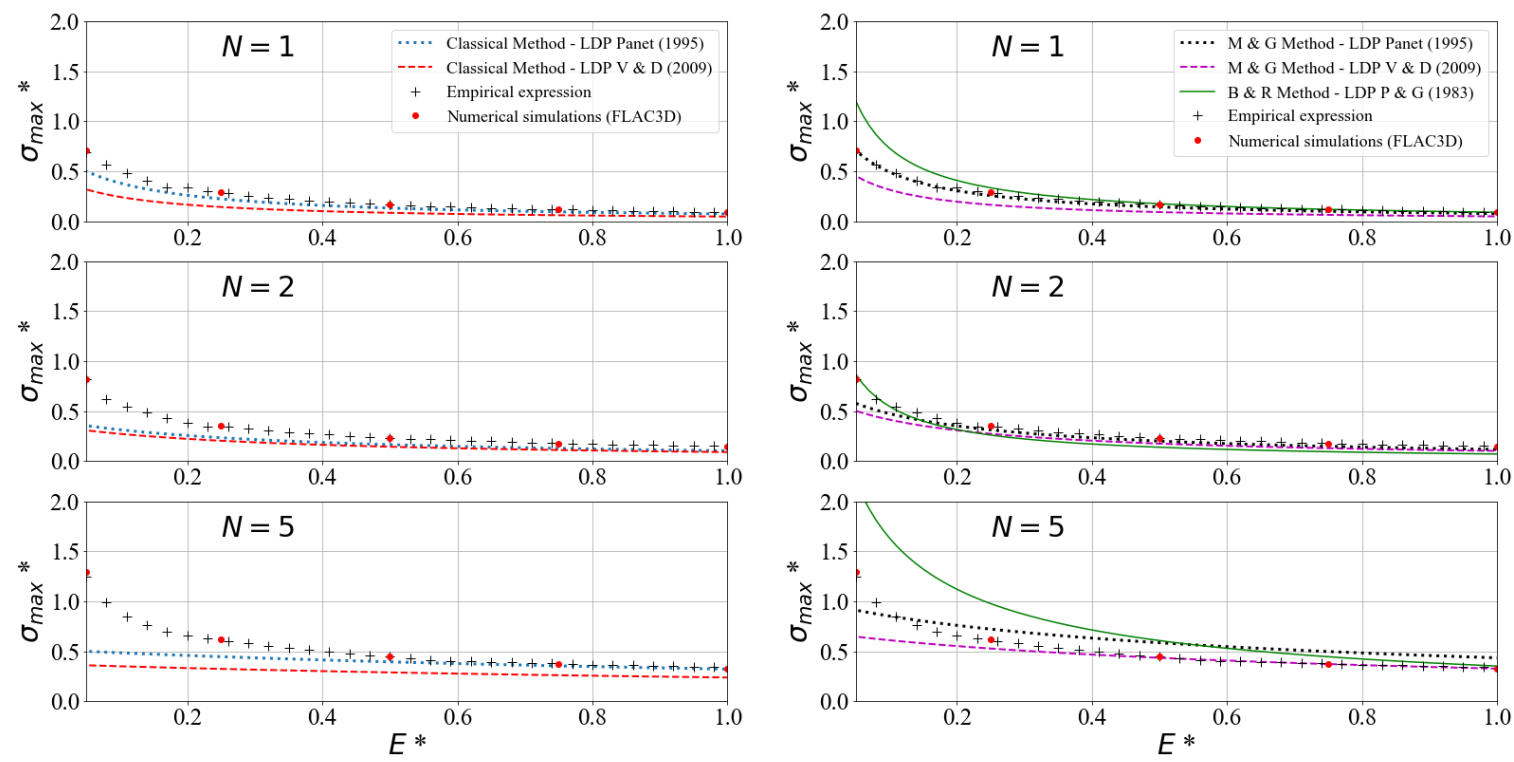

Fig6. Comparison of $\sigma_{\max }{ }^{*}$ between the different approaches when $R^{*}=12.5$ and $\phi=25^{\circ}$ if $\psi$ is equal to $\phi / 3$. Classical methods on the left column and implicit methods on the right 

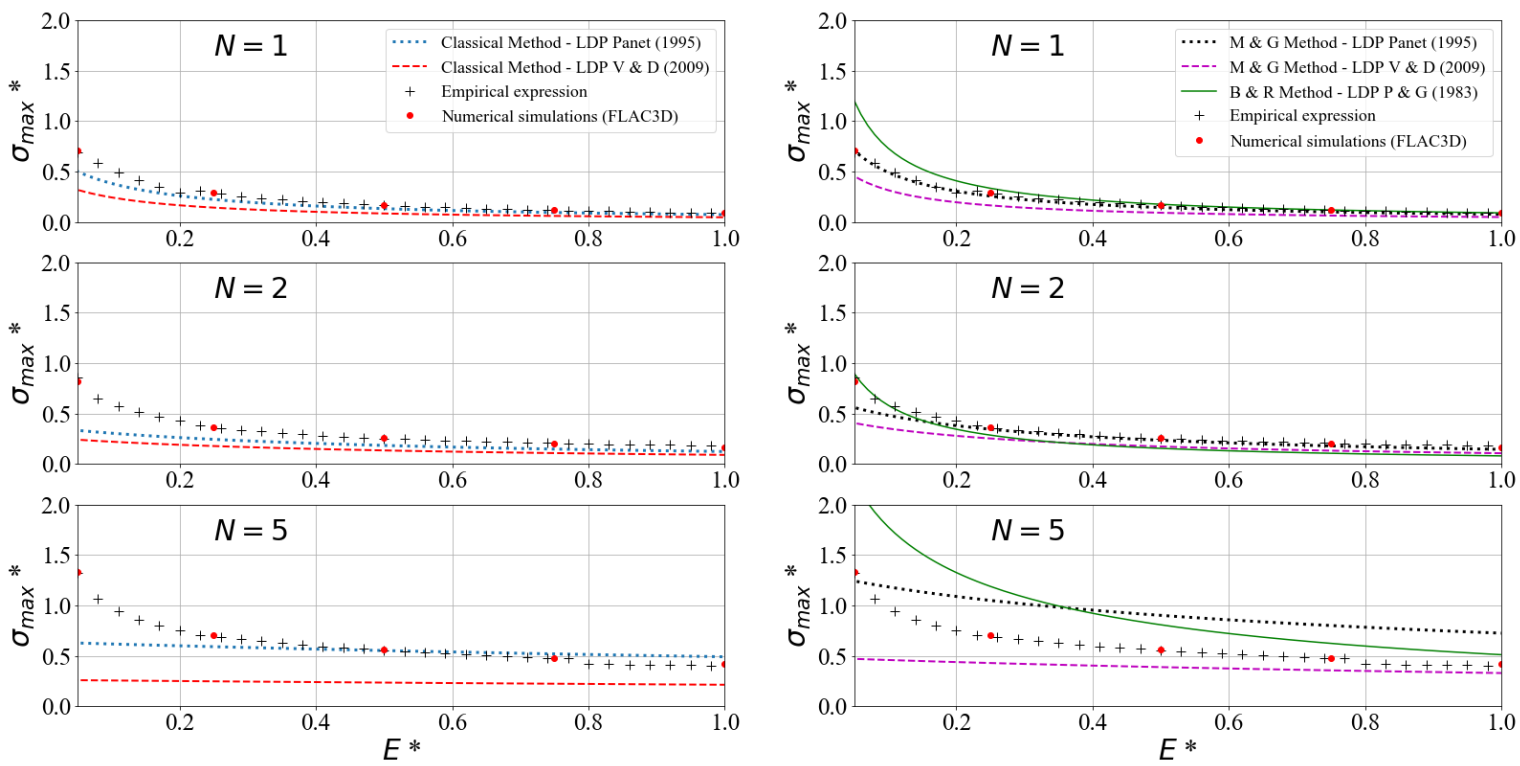

Fig7. Comparison of $\sigma_{\max }{ }^{*}$ between the different approaches when $R^{*}=12.5$ and $\phi=25^{\circ}$ if $\psi$ is equal to $\phi$. Classical methods on the left column and implicit methods on the right column 

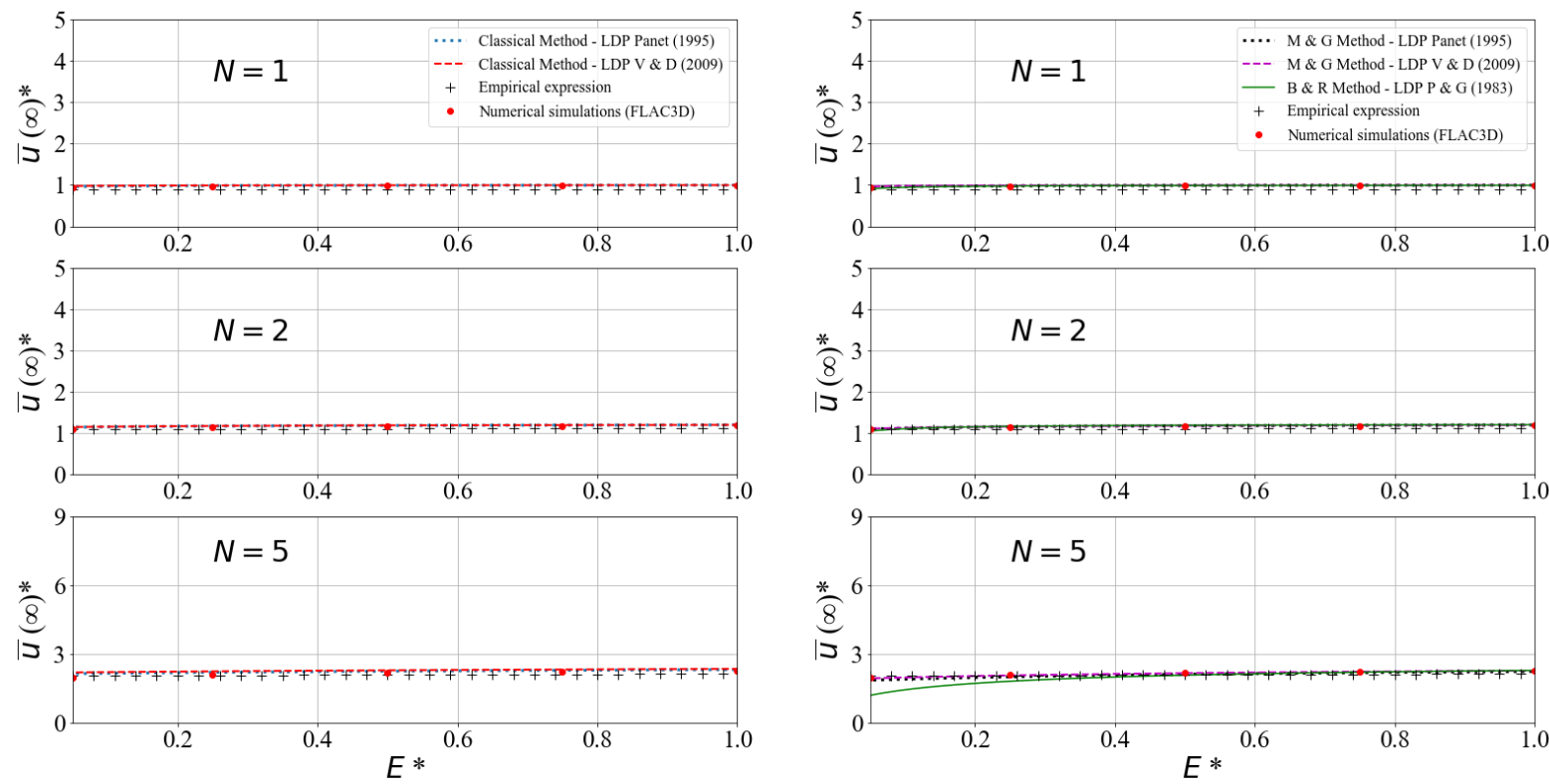

Fig8. Comparison of $\bar{u}(\infty)^{*}$ between the different approaches when $R^{*}=12.5$ and $\phi=25^{\circ}$ if $\psi$ is equal to 0 . Classical methods on the left column and implicit methods on the right column 

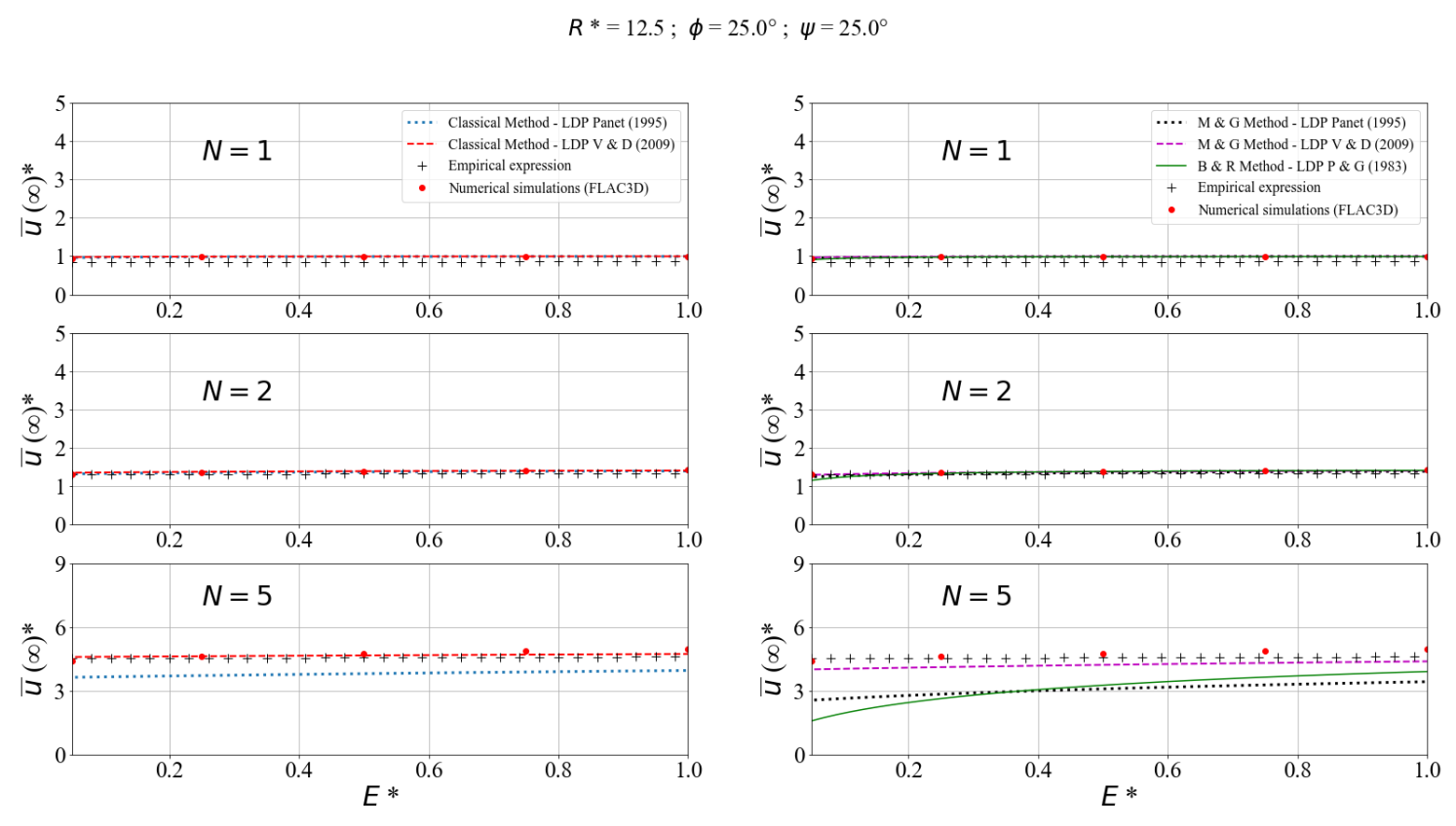

Fig9. Comparison of $\bar{u}(\infty)^{*}$ between the different approaches when $R^{*}=12.5$ and $\phi=25^{\circ}$ if $\psi$ is equal to $\phi$. Classical methods on the left column and implicit methods on the right column 


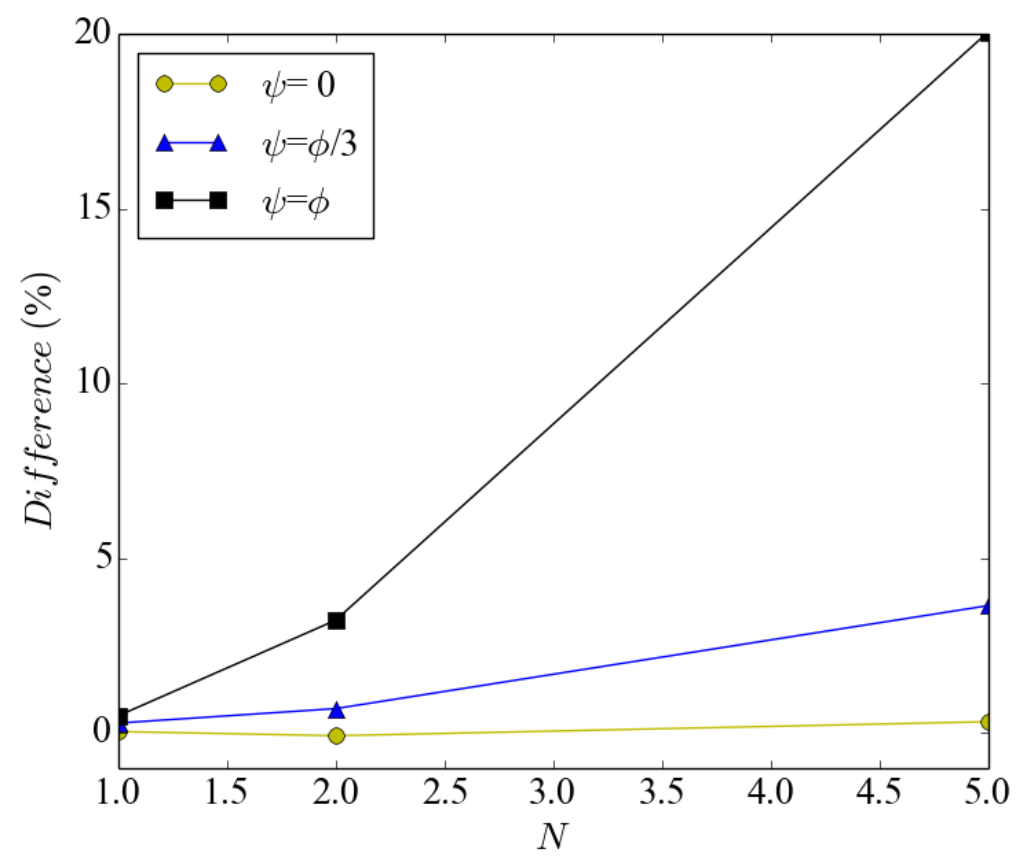

Fig10. Effect of $N$ on the difference between the numerical simulation and the plane strain assumption $\left(\phi=35^{\circ}\right.$ and $\left.v=0.25\right)$ 


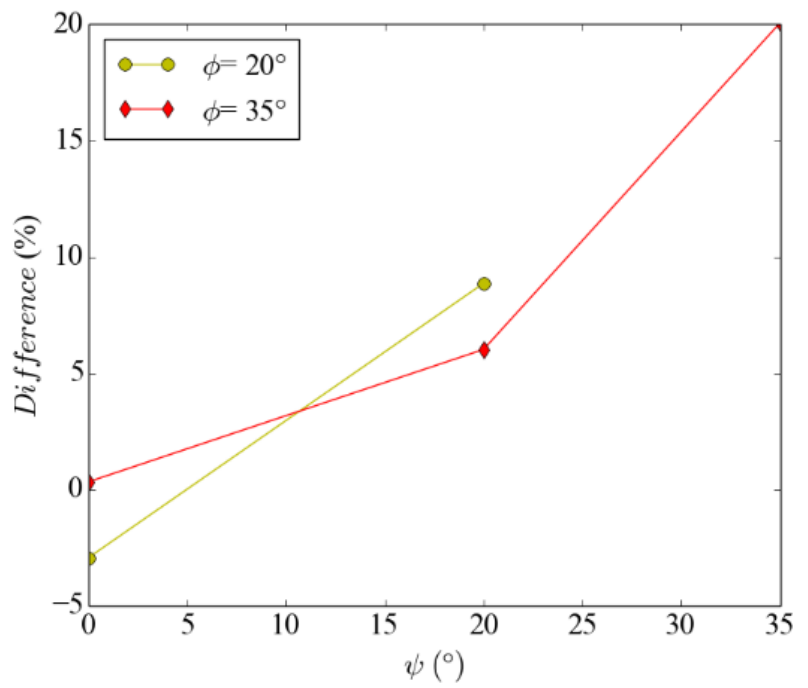

Fig11. Effect of $\phi$ on the difference between the numerical simulation and the plane strain assumption $(N=5$ and $v=0.25)$ 

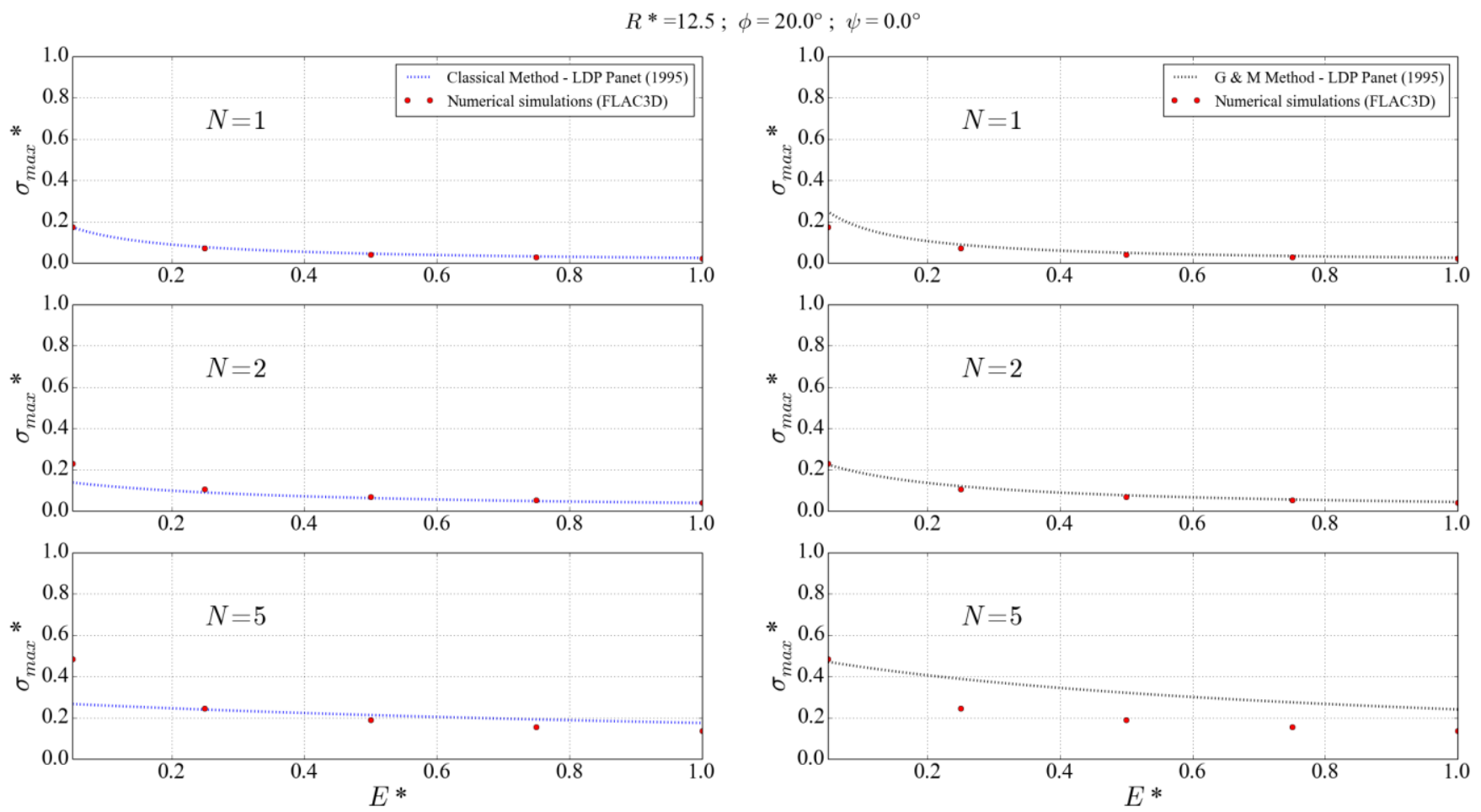

Fig12. Comparison of $\sigma_{\max }{ }^{*}$ between the different approaches when $d^{*}=2, R^{*}=12.5$ and $\phi=20^{\circ}$ for incompressible plasticity $(\psi=0)$. Classical method on the left column and implicit method of Nguyen-Minh \& Guo on the right column 

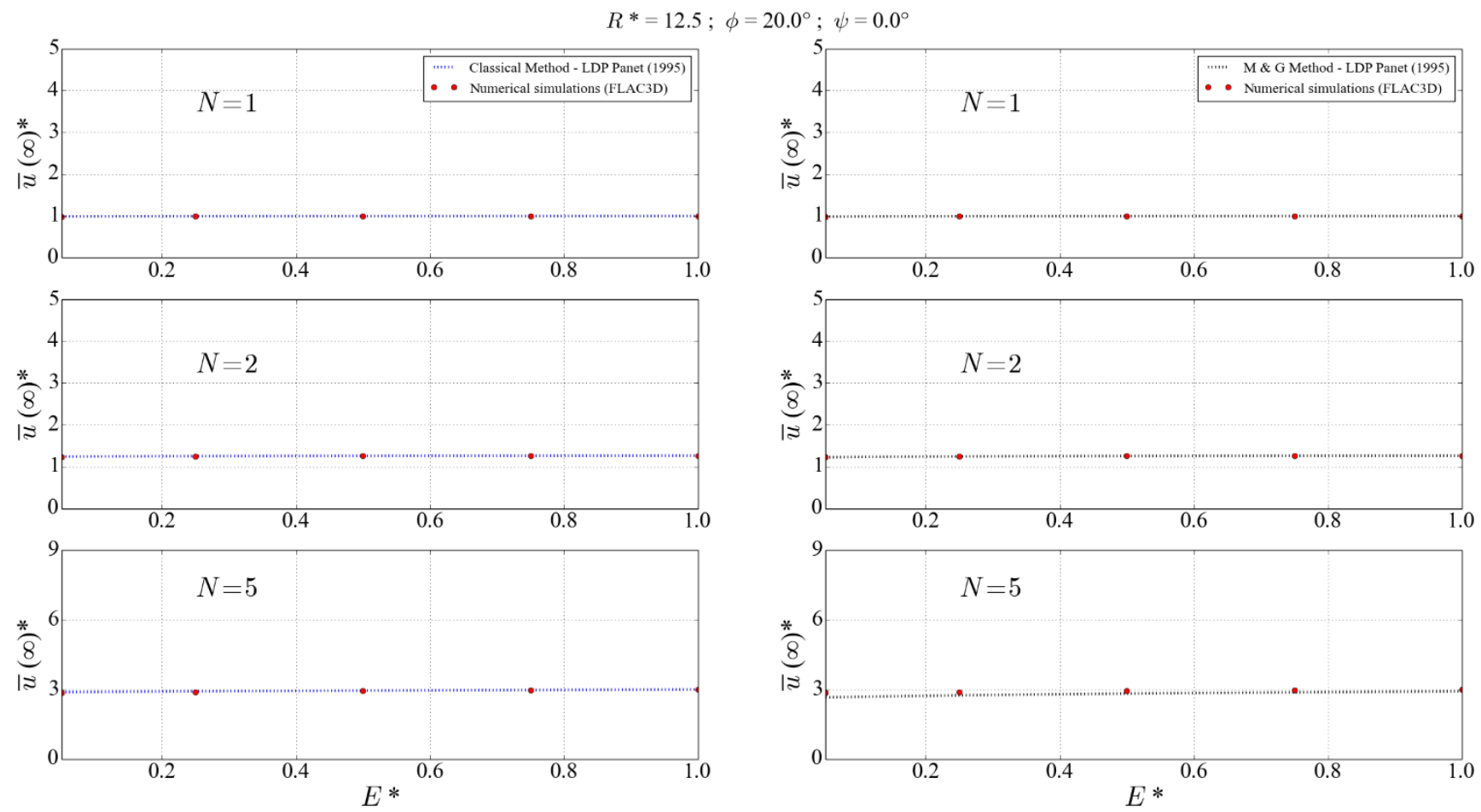

Fig13. Comparison of $\bar{u}(\infty)^{*}$ between the different approaches when $d^{*}=2, R^{*}=12.5$ and $\phi=25^{\circ}$ for incompressible plasticity $(\psi=0)$. Classical method on the left column and implicit method of Nguyen-Minh \& Guo on the right column 